\title{
Fast ignition schemes for inertial confinement fusion
}

\author{
C. Deutsch ${ }^{\mathrm{a}}$ \\ LPGP (UME-CNRS 8578), Bât. 210, UPS, 91405 Orsay Cedex, France
}

Received: 8 January 2002 / Received in final form: 17 January 2003 / Accepted: 12 June 2003

Published online: 20 October 2003 - (C) EDP Sciences

\begin{abstract}
The controlled production of a local hot spot in supercompressed deuterium + tritium fuel is examined in details. Relativistic electron beams (REB) in the $\mathrm{MeV}$ and proton beams in the few tens $\mathrm{MeV}$ energy range produced by PW-lasers are respectively considered. A strong emphasis is given to the propagation issues due to large density gradients in the outer core of compressed fuel. A specific attention is also paid to the final and complete particle stopping resulting in hot spot generation as well as to the interplay of collective $v s$. particle stopping at the entrance channel on the low density side in plasma target. Moreover, REB production and fast acceleration mechanisms are also given their due attention. Proton fast ignition looks promising as well as the wedged (cone angle) approach circumventing most of transport uncertainties between critical layer and hot spot. Global engineering perspectives for fast ignition scenario (FIS) driven inertial confinement fusion are also detailed.
\end{abstract}

PACS. 52.40.Mj Particle beam interactions in plasmas - 52.35.Py Macroinstabilities (hydromagnetic, e.g., kink, fire-hose, mirror, ballooning, tearing, trapped-particle, flute, Rayleigh-Taylor, etc.) 34.50.Bw Energy loss and stopping power

\section{Ignition vs. compression}

Up to now, it has been impossible to obtain simultaneously the compression and the heating of a DT target because of problems related to the global efficiency of the compression. In particular, this is limited by implosion asymmetries. Those arise from the defects of the capsule manufacture, the quality of its surface finish and the nonuniformity in space and time of the laser irradiation. With a maximum acceptable defect of the final radius $R_{f}$ of the pellet of about $33 \%$ [1] we obtain $\delta R_{f} / R_{f}=C(\delta v / v) \leq 1 / 3$. Here $v$ is the implosion speed and $C=R_{i} / R_{f}$ the convergence ratio (ratio of the initial to the final radius of the pellet). A typical value of $C=30$ is expected, so that the required uniformity of the implosion velocity must be better than $1 \%$.

The small initial perturbations also give rise to different hydrodynamic instabilities, which contribute strongly to breaking down the implosion symmetry. The most important ones, the Rayleigh-Taylor instabilities [2] and the Richtmyer-Meshkov instabilities [3], grow at the interface between fluids of different densities. The Rayleigh-Taylor instabilities arise typically at the ablation front of the pellet during the acceleration phase and at the shell/fuel interface during the deceleration phase. During the intermediate stage, the Richtmyer-Meshkov instabilities, induced by the shock wave emerging from the inner surface of the

\footnotetext{
a e-mail: claude.deutsch@lpgp.u-psud.fr
}

shell, appear. The global effect of these instabilities is to enhance the initial perturbations until the ablator material pollutes the fuel mixture. The consequence is an important decrease of the temperature and a degradation of the compression. It can be shown that [4] the hydrodynamic instabilities become more important as the "aspect ratio" $R / \Delta R$ of the capsule is increased. A value of $R / \Delta R=25-35$, with typically $\Delta R=70-100 \mu \mathrm{m}[5]$ would represent a good compromise in order to get an adequate compression.

At present, ignition has not yet been experimentally achieved, and implosion experiments have only produced either high compression, or high temperature. The maximum density reached was 600 times liquid density [6], corresponding to $\rho R \approx 0.1 \mathrm{~g} / \mathrm{cm}^{2}$. During this experiment, the maximum temperature was not larger than $300 \mathrm{eV}$, insufficient to produce an appreciable number of fusion reactions. The maximum neutron yield, $2 \times 10^{14}$ for a temperature of $15 \mathrm{keV}$, has been obtained for a density of only $2 \mathrm{~g} / \mathrm{cm}^{3}$ corresponding to a convergence ratio of 4 [7]. Those results motivate, a new generation of laser facilities, including ignition with the "classic" scheme just described, and a greater energy, is under development to achieve ignition [1].

Recently, the rapid development of short-pulse laser beam technology has allowed an alternative approach to ICF: the Fast Ignitor (FI) [8]. The idea is to dissociate the fuel ignition phase from its compression. The fuel is 

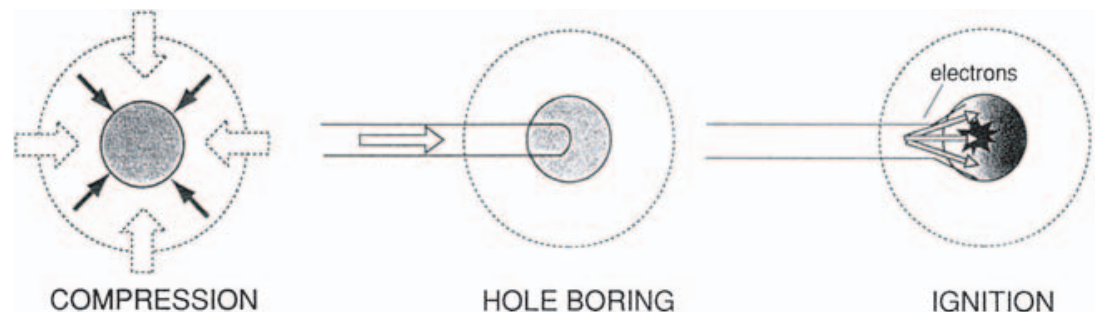

Fig. 1. The three phases characterising the fast ignitor scheme. A suprathermal electron beam generated in the interaction with an ultra-intense laser brings the supercompressed fuel to ignition.

previously compressed as in the "traditional" scheme and then brought to ignition by means of an external energy sources: a short high-intensity laser beam focused on the side of the pellet.

The scheme is based on three stages $[8,9]$ (see Fig. 1). At the moment of maximum compression (the "classic" implosion phase) a first short-pulse laser beam $(\tau \approx$ $100 \mathrm{ps})$ delivering an intensity of $10^{19} \mathrm{~W} / \mathrm{cm}^{2}$ bores a channel $\left(n<n_{c}\right)$ in the ablated part of the capsule ("hole boring" phase), by virtue of interaction mechanisms typical of the high-intensity laser regime [10]. A second shorter $(\tau \approx 10 \mathrm{ps})$ and more intense $\left(10^{20} \mathrm{~W} / \mathrm{cm}^{2}\right)$ laser beam, aligned with the first one, can propagate in the channel towards the high-density regions of the fuel. The rapid transfer of laser energy to the compressed fuel occurs via very energetic electrons, to so-called Suprathermal or Fast electrons. The generation of fast electrons is particularly important in the case of the high-flux regime. These electrons are supposed to propagate up to the core of the compressed capsule and deposit their energy. This rapid heating creates temperatures needed to turn on the fusion reactions in a lateral, but not central, hot spot in the fuel.

The difference between the two possible approaches for hot spot generation and the achievement of ignition is shown schematically in Figure 2. In the "classic" case, from the beginning, the DT mass needed for the hot spot formation is brought to a high temperature by the converging shocks, while a slow implosion compresses the rest of the fuel. This leaves the two parts of the fuel at different temperatures. In the case of the fast ignitor, all of the fuel is compressed to a large density, so at the end of the implosion, a fraction of the DT is heated by an external source (the ultra-intense laser) to form the hot spot. The characteristic profiles of the thermodynamic quantities in the hot spot and in the main fuel are also shown in Figure 2 ; and isobaric [11] or isochoric [12] compression model are respectively used to describe the behaviour of the fuel during the ignition.

The fast ignitor presents distinct advantages in comparison with the usual scheme, and offers the possibility of obtaining ignition with current technological means. The expected heating is caused by an external element (the fast electron beam) on a very short time scale, much shorter than the hydrodynamic motion of the capsule. The ignition does depend neither on the simultaneous presence of the hot spot, for starting the reaction, nor on the surrounding colder compressed fuel, for the burn wave gen-
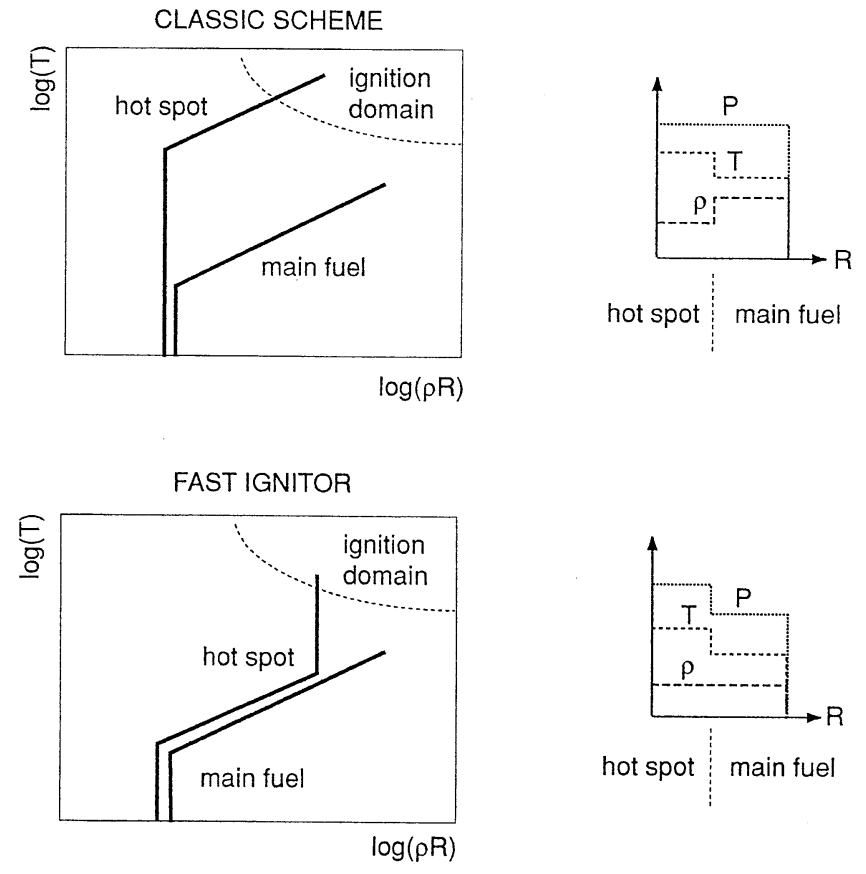

Fig. 2. Principle of the hot spot generation and of fuel ignition in the 'classic' scheme and in the 'fast ignitor'.

eration; this being feasible, in fact, only with a "perfect" implosion. It is hence possible to reduce significantly the constraints on the implosion symmetry which usually limit compression and heating efficiency.

At the same time, the density constraints are also reduced in comparison with those implied by the isobaric model to maintain the pressure equilibrium of the fuel. This will give a higher energy gain because less energy is used to reach high densities. More recent calculations, although less optimistic than the initial ones proposed by Tabak et al., show that, for a laser energy of about $1 \mathrm{MJ}$, the isochoric gain is nearly three time greater than the isobaric one [13].

The success of the fast ignitor scheme nevertheless depends on the electron source characteristics. The electron beam must be 'piercing enough' to propagate in the most compressed parts of the fuel, where the density reaches $200-400 \mathrm{~g} / \mathrm{cm}^{3}$. At the same time, the energy deposition must be quite local (typically $10 \mu \mathrm{m}$ ) in order to form a hot spot small enough for significant heating. Moreover, the electrons must be quite energetic to heat this fuel 
fraction to ignition temperature. According to recent calculations [14], an electron beam with a characteristic temperature of $1 \mathrm{MeV}$, should have a penetration distance of around $10 \mu \mathrm{m}$ in a fuel of density $300 \mathrm{~g} / \mathrm{cm}^{3}$. The energy required to heat at $10 \mathrm{keV}$ a mass of DT with these dimensions is about $10 \mathrm{~kJ}$, corresponding to a number of electrons of $10^{16}-10^{17}$. Assuming a typical conversion efficiency of laser energy into fast electrons of $10-20 \%$, a laser delivering $100 \mathrm{~kJ}$ in $10 \mathrm{ps}$, which is technologically conceivable is therefore required to produce such an electron source.

More generally, the described scenario involves different aspects concerning laser-plasma interaction physics in the high-flux case, a subject of intense research all around the world, both on the theoretical as well as on the experimental level. The difficulties related to the achievement of ignition are then transferred into those concerning the propagation and coupling with matter of high-intensity short-pulse lasers. The feasibility of such a project demands a full knowledge of the implied processes, which can be summarised as:

- Channel boring in the ablated part of the capsule.

- Fast electrons generation and characterisation of the acceleration process.

- Energy transfer mechanisms of those electrons to the compressed fuel.

\section{Channel boring}

For the past several years, electromagnetic irradiances above $10^{18} \mathrm{~W} / \mathrm{cm}^{2}$ have been achieved by focusing the picosecond light pulses delivered by compact multiterawatt lasers operating at micrometer wavelength [15]. The associated electric field, which is higher than the electric field existing in the Bohr atom, can then instantaneously transform the matter into a plasma. Moreover, it lets the free electrons quiver with relativistic momentum; the latter normalized to $m_{e} c$, is indeed written as $a_{0}=0.85\left(I_{0} \lambda_{0}^{2} / 10^{18}\right)^{1 / 2}$, where $m_{e}$ is the electron mass, $c$ is the light velocity in vacuum, $\lambda_{0}$ is the laser wavelength in units of $\mu \mathrm{m}$, and $I_{0}$ is the laser irradiance in units of $\mathrm{W} / \mathrm{cm}^{2}$. It has been predicted that high irradiances, by increasing the inertial electron mass and hence decreasing the effective plasma frequency, will allow a laser to penetrate a "classically opaque" plasma.

In the standard view of laser-plasma interaction, a laser wave with wavelength $\lambda_{0}$ that impinges on a plasma with electron density above $n_{c}=10^{21} / \lambda_{0}^{2}$ in units of $\mathrm{cm}^{-3}$ is partially reflected and partially absorbed by the socalled normal skin effect, resulting from the electron-ion collisions inside the wave penetration depth $c / \sqrt{\omega_{p e}^{2}-\omega_{0}^{2}}$; $\omega_{p e}$ and $\omega_{0}$ denote the electron plasma frequency and the incident radial frequency, respectively. High electron quiver velocities result in reduced collisions and the anomalous skin effect. Particle-in-cell (PIC) simulations have clearly exhibited collisionless absorption via kinetic effects: resonant absorption and vacuum screening effect for oblique incidence [16], magnetic force $\mathbf{j} \times \mathbf{B}$, for normal incidence generates fast electron bursts entering the solid target. PIC simulations revealed hydrodynamic scenarios, namely, plasma pushing, hole boring, and electrostatic shock [17].

Recent results by from $1 \frac{1}{2} \mathrm{D}$ relativistic PIC simulations that evidence a transition between an opacity regime (OR) and a transparency regime (TR), respectively characterized by the absence or the propagation of the laser wave inside the plasma (Lefebvre-Bonnaud [16]). The simulations were performed using the code Euterpe, with singly ionized mobile ions with mass $m_{i}=1836 m_{e}$, unless otherwise specified. The plasma is assumed to be electrically neutral, with an initial temperature of $1 \mathrm{keV}$.

The differences between OR and TR are best illustrated by the electron trajectories displayed in Figure 3 for (a) OR and (b) TR at density $n_{e} / n_{c}=2$. In the first case, the laser wave does not penetrate the plasma but pushes its surface, resulting in a large density increase and a laser reflectivity $R \approx 1$. In contrast, the plasma edge remains nearly stationary in the second case, while the laser propagates deeper and deeper in the target, setting the electrons in violent motion. Both pictures show a surface recoiling at steady velocity. When irradiance is increased, this velocity $\beta$ exhibits the following features (Fig. 4a): (1) a slow linear increase corresponding to OR, (2) a jump for a critical laser strength $a_{0 e}$ (that increases with $n_{e}$ ), and (3) a rapid increase in TR. The frequency $\omega_{r}$ of the light reflected by the plasma displays opposite trends (Fig. 4b).

The critical laser amplitude $a_{0 c}$ at which the transition between OR and TR occurs can be determined from the $\beta$ or $\omega_{r}$ plots, but more precisely from the electron trajectories. Indeed, near $a_{0 c}$, the transient penetration of the laser wave can be observed, during which the ions are nearly immobile. Then an ion shock forms, which values of $\beta$ and $\omega_{r}$ are provided.

The laser electric field is expected to penetrate easily a plasma interface. Such an effect is well documented numerically by the laser squared electric field into a plasma slab with density $n_{e}=1.5 n_{c}$ at $0.1 \mathrm{ps}$ after the impulsion start. Then, in Figure 5, Adam depicts such a perforating process for a laser Gaussian profile, with $2.44 a_{0}$ diameter and intensity $2.45 I_{0}$. All these numerical evidences highlight pretty convincingly the relativistic crushing of the plasma critical surface (Fig. 6).

\section{Fast electron generation and acceleration}

Several mechanisms are thought to be simultaneously at work to produce and accelerate relativistic electrons in the $\mathrm{MeV}$ energy range [15].

Here, we shall emphasize the most conspicuous one, the ponderomotive force.

The target electron escapes the laser pulse reflected at critical density (see Fig. 7), with a kinetic energy equal to oscillating energy on one arch of laser pulse. It is then forwardly ejected through the Lorentz force $\mathbf{V} \times \mathbf{B}$. 

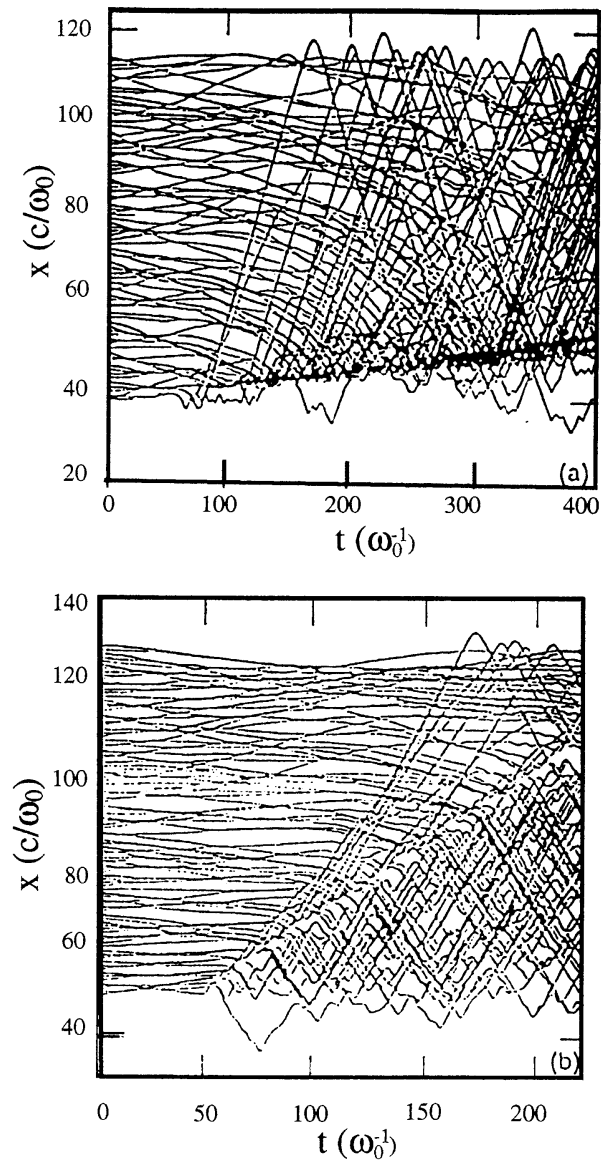

Fig. 3. Electron trajectories for $n_{e} / n_{c}=2, m_{i} / m_{e}=1836$, and also $a_{0}=2$ (a) or $a_{0}=4$ (b) [16].

More specifically, one might be asking for suitable processes explaining $\mathrm{MeV}$ electron energies in the given interactions.

According to Lawson-Woodward theorem [18]; no net energy transfer from EM wave to electrons possible if:

1. the laser fields are in vacuum;

2. the electron is relativistic;

3. no static $E$ or $B$ fields are present;

4. the region of interaction is infinite;

5. Nonlinear forces (ponderomotive) are absent.

However, we are violating 1,2,3,4 and 5!

So, electrons interacting with EM wave in a plasma can break Lawson-Woodward theorem, thus allowing very energetic electrons to be generated.

One of the major physics issues to implement fast ignition is the generation and propagation of energetic hot electrons in high-density plasmas. Intensive 2D PIC simulations due to Adam et al. [17] demonstrate that parametric instabilities could help the relativistic electrons to penetrate indepth a dense target. Recently, the energetic hot electrons outgoing from plasmas were studied experimentally and theoretically [19-24]. Hot-electron energies up to $100 \mathrm{MeV}$ were observed in laser-solid interactions at an intensity of $3 \times 10^{20} \mathrm{~W} / \mathrm{cm}^{2}$ using petawatt laser system at LLNL [25]. Malka and Miquel also studied rel-

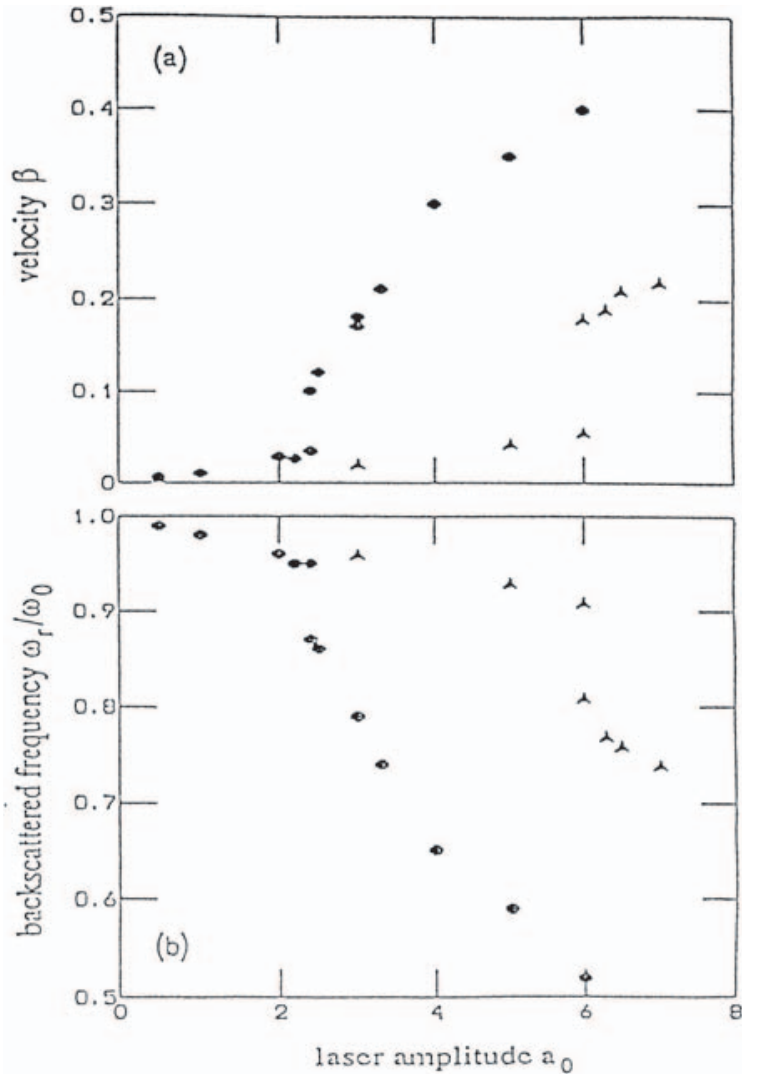

Fig. 4. (a) Velocity of the interface (in units of $c$ ) and (b) frequency of the reflected light (in units of $\omega_{0}$ ) as a function of the laser amplitude $a_{0}$, for $m_{i} / m_{e}=1836$ and $n_{e} / n_{c}=2(\bullet)$ or 4 (E. Lefebvre and G. Bonnaud, in Ref. [16]).

ativistic electrons produced by interaction of relativistically intense laser pulses with solid targets [26]. The high energy of hot electrons was attributed to $\mathbf{J} \times \mathbf{B}$ acceleration mechanism [27]. On the other hand, hot electrons ingoing along the laser propagation direction, which is directly related with fast ignition, were also studied in detail $[28,29]$. Tatarakis et al. observed a plasma at the back surface of a thin foil. This was believed to be formed by a collimated electron beam passing through the foil [30]. A more direct experiment was carried out using a transparent glass target coated with an aluminum layer. An ionization track induced by a beam of energetic hot electrons was clearly observed through the target [31,32]. Norreys et al. observed a highly directional $\gamma$-ray beam with energies above $10 \mathrm{MeV}$ in the direction nearly opposite to the target normal direction. This indicated the generation of a directional relativistic electron beam [33]. These experimental results imply that the hot-electrons could be collimated by self-generated magnetic fields. The generation of hot-electron jet and the pinching of magnetic fields were reproduced by particle-in-cell (PIC) simulations [34,35].

Many mechanisms can produce hot electrons. In the interaction of intense laser pulses with sharp solidvacuum interface, the ponderomotive potential model and Brunel absorption model [36] play important roles. Other 


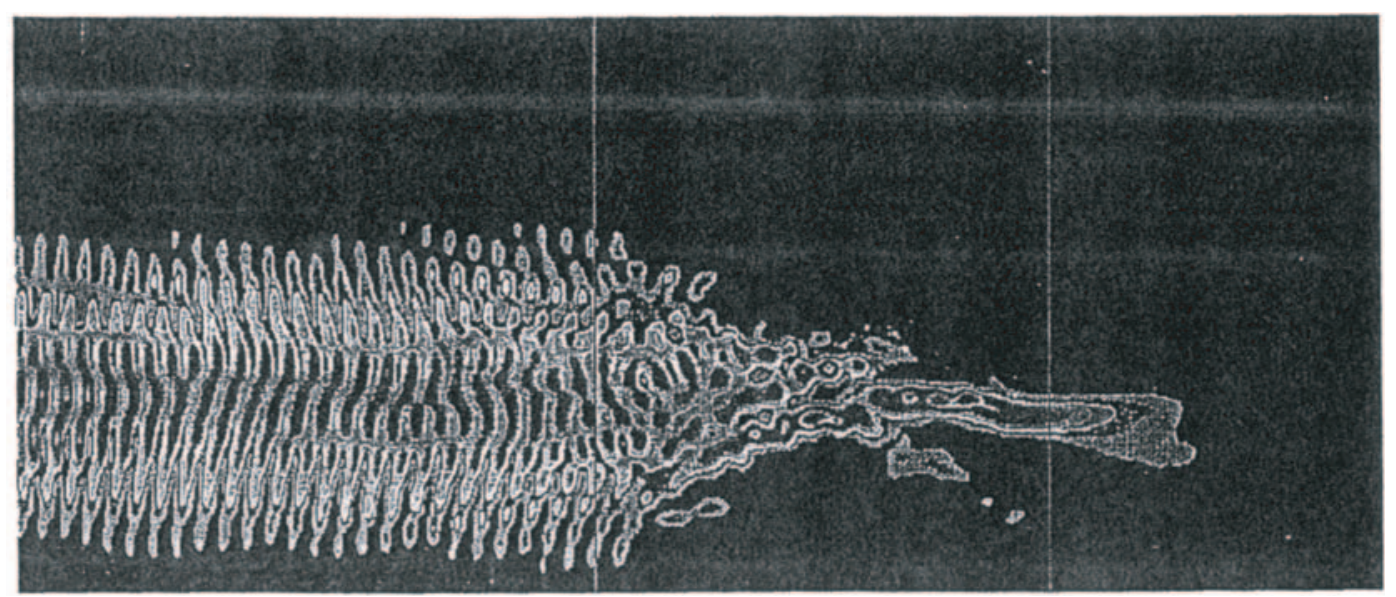

Fig. 5. Laser wave $|\mathbf{E}|^{2}$ in a plasma layer at density 1.5 critical, 0.1 ps after pulse start (J.C. Adam, loc. cit. in Ref. [57]).

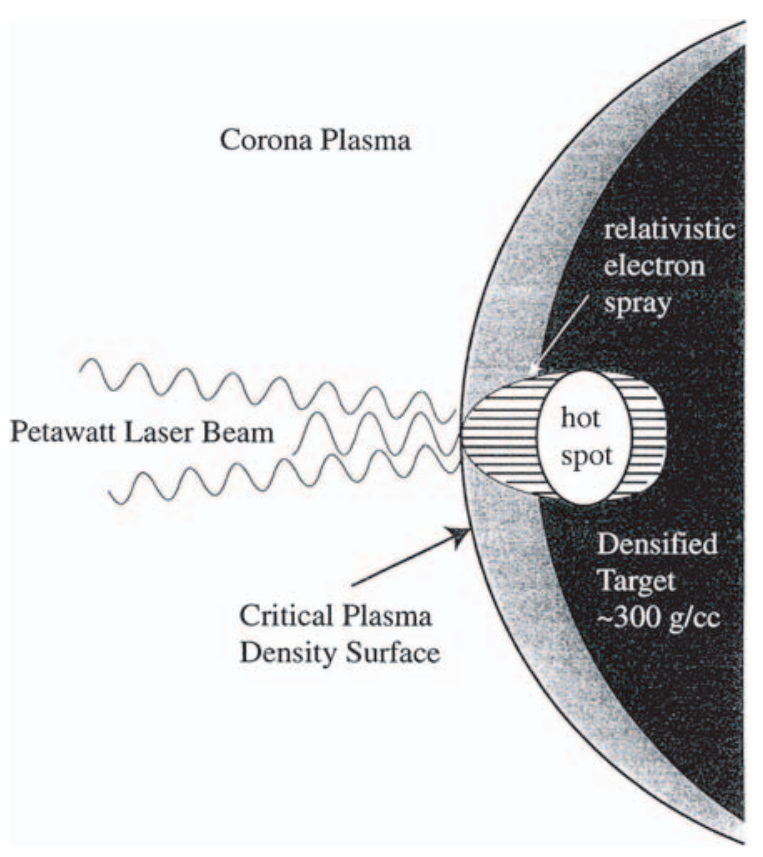

Fig. 6. Complete scheme for fast ignition with hole boring in the outer layers of the DT precompressed core.
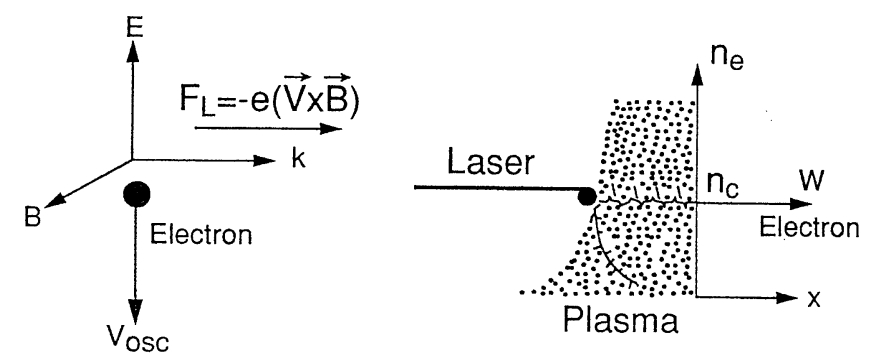

Fig. 7. Forward electron acceleration.

mechanisms such as wake fields [37], parametric instabilities [38,39]. B-loop mechanism [40], and propagating wave [41] are effective when a large scale preplasma is formed before the arrival of the main laser-beam.

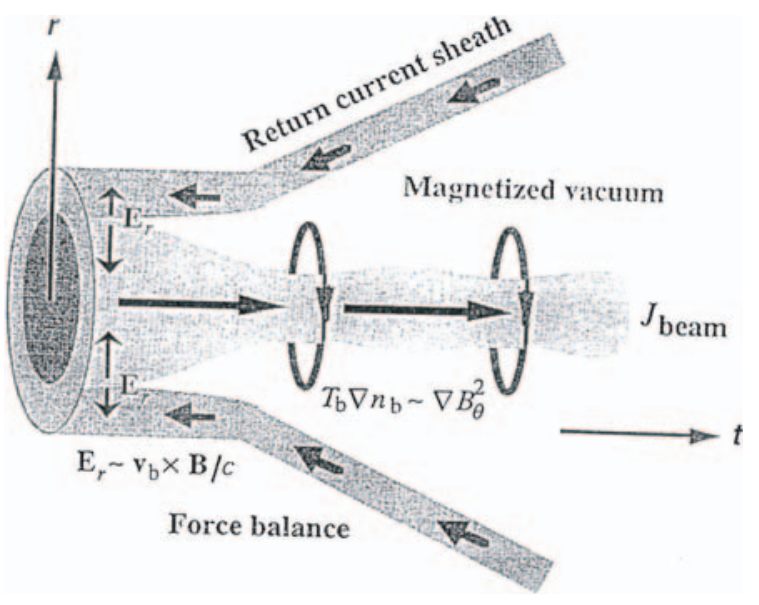

Fig. 8. Schematic view illustrating the formation of a coaxial beam channel. For explanations see text (A. Pukhov, J. Meyerter-Vehn, in Ref. [40]).

Magnetic field assisted particle acceleration ("B-loop" mechanism) relies essentially on electron gaining energy from EM wave similar to vacuum case, but due to the presence of the azimuthal B-field, electron is "dephased" in EM wave, and thus gains more energy than it otherwise would have.

Trapping and acceleration of the background electrons can result from the coupling of Raman backscattering to the wakefield (threshold much lower than even the cold 1-D wave breaking limit).

In order to trigger out efficiently the hot spots at the end of range of the laser produced relativistic electron beams (REB) through kJ of local energy deposit, one has to envision e-beams with pretty large number densities $n_{b} \sim 10^{22} \mathrm{~cm}^{-3}$.

Obviously, the given REB have to be propagated charge- and current-neutralized to overcome the rather restrictive Alfven-Lawson limit [42] $I \leq I_{A L}=17 \beta \gamma \mathrm{kAmp}$, when $\beta$ and $\gamma$ are the usual relativistic parameters in terms of overall REB velocity $V_{b}$. So, one expects a return current (Fig. 8) to compensate for the incoming REB one. 


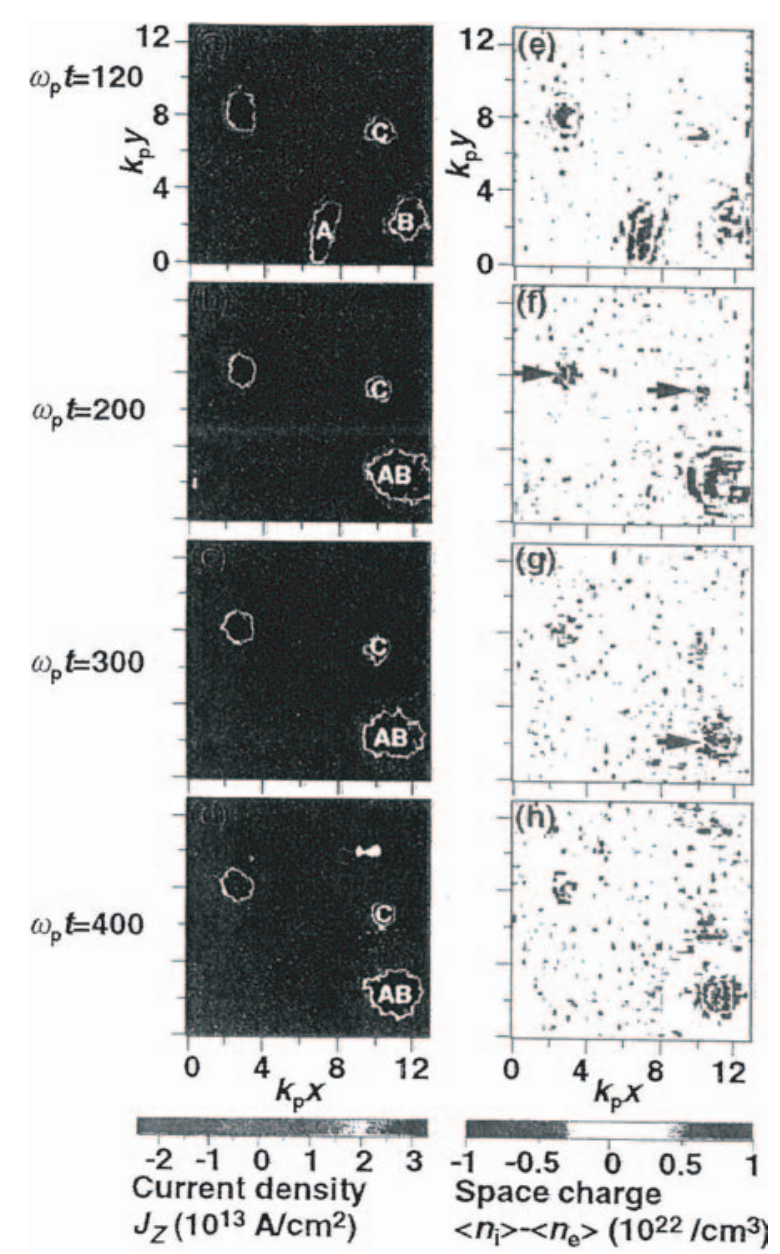

Fig. 9. Left column: $J_{z}$ current density plotted in transverse $(x, y)$ plane for times given, right column: corresponding space charge density, given for times $\omega_{p t}=1220(\mathrm{a}, \mathrm{e}), 200(\mathrm{~b}, \mathrm{f})$, $300(\mathrm{c}, \mathrm{g})$, and $400(\mathrm{~d}, \mathrm{~h})$. Arrows mark spots of imploded ion cores (Honda et al., Ref. [44]).

The corresponding dynamic picture depicts an anisotropic velocity distribution. So, one could reasonably expect a strong transverse and electromagnetic interaction between the two counterstreaming currents leading to the well-known Weibel EM instability (WEI) [43]. Very recently, some authors have thus been inclined [44] to deduce from very heavy PIC simulations with quasiparticle number $\geq 10^{8}$ but in rather small simulation boxes $(4 \mu \mathrm{m} \times 4 \mu \mathrm{m} \times 8 \mu \mathrm{m})$ that WEI could very rapidly turn nonlinear. So, a highly destructive filamentation (for instance, Figs. 9 and 10) of the incoming REB could rapidly occur, thus preventing any further controlled propagation toward the DT compressed core.

Actually, it should be emphasized out that the gradient effects due to too close boundaries in the given simulation boxes may be describing a physics picture different from the one at stake, here.

To clear up those controversies, we considered REB propagation in a smooth target density gradient (Fig. 11) mimiking penetration in the outer layers of DT precompressed core. Then, we paid attention to the ini-

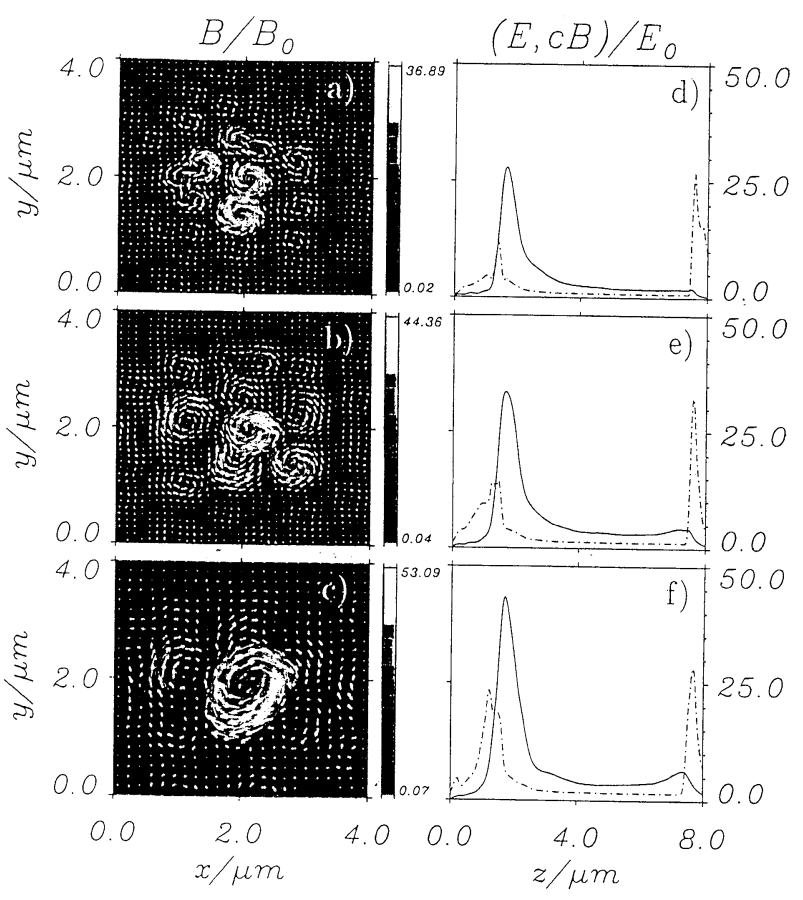

Fig. 10. Merging of magnetic filaments and cycle-averaged $E$ and $B$ fields versus $z$ in units $E_{0}$ and $B_{0}$. Plots (a, b, c) show the planes $z=2.0 \mu \mathrm{m}$ of the cycle-averaged magnetic field $B=\left(B_{x}^{2}+B_{y}^{2}+B_{z}^{2}\right)^{0.5}$ at different times. Plots (d, e, f) show $E=\int d x d y\left(E_{x}^{2}+E_{y}^{2}+E_{z}^{2}\right)^{0.5}$ (dashed) and $B=\int d x d y\left(B_{x}^{2}+\right.$ $\left.B_{y}^{2}+B_{z}^{2}\right)^{0.5}$ (solid) averaged over the lateral area and a laser cycle. The times are $t=71 \mathrm{fs}(\mathrm{a}, \mathrm{d}), t=125 \mathrm{fs}$ (b, e) and $t=179 \mathrm{fs}(\mathrm{c}, \mathrm{f})$. The arrows in (a, b, c) point clockwise. They indicate the direction of the cycle-averaged magnetic field $B$. The normalization parameters are $E_{0}=1.0 \times 10^{11} \mathrm{~V} \mathrm{~m}^{-1}$ and $B_{0}=8.76 \times 10^{2} \mathrm{Vs} \mathrm{m}^{-2}$. [H. Ruhl et al., Plasma Source, Sci. Technol. 11, A-154 (2002).]

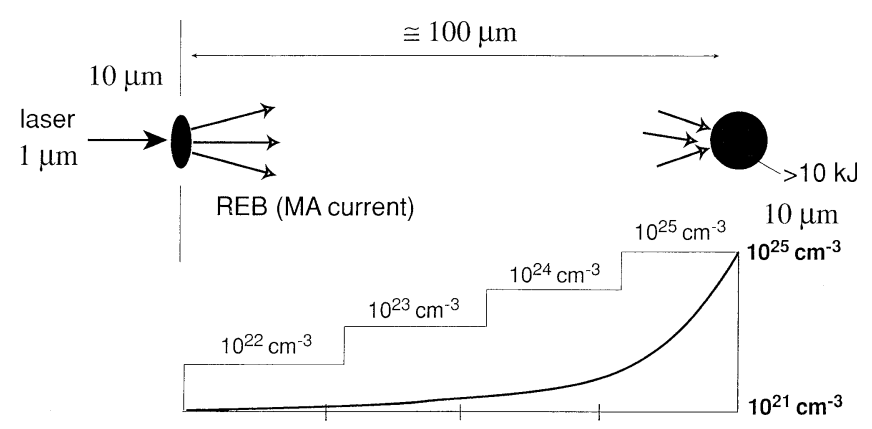

Fig. 11. Relativistic electron beam (REB) propagation with $\mathrm{MeV}$ incoming energy through layers of increasing density $N_{p}$ in a core of precompressed DT fuel.

tial linear growth rate of WEI. Typically, we took REB particle energy $E_{b}=1 \mathrm{MeV}$ with isotropic temperatures $T_{b}=1 \mathrm{keV}$ and $T_{P}($ target $)=100 \mathrm{eV}$, respectively. Connecting plain linear growth with mode-mode coupling theory, we got profiles for WEI quasi-linear growth rate $\delta$ denoted as weakly turbulent.

Thus, one can check that although $\delta / \omega_{p}$ significantly decreases with increasing target plasma density $N_{p}, \delta$ remains nearly constant (at best). So, linear WEI growth 


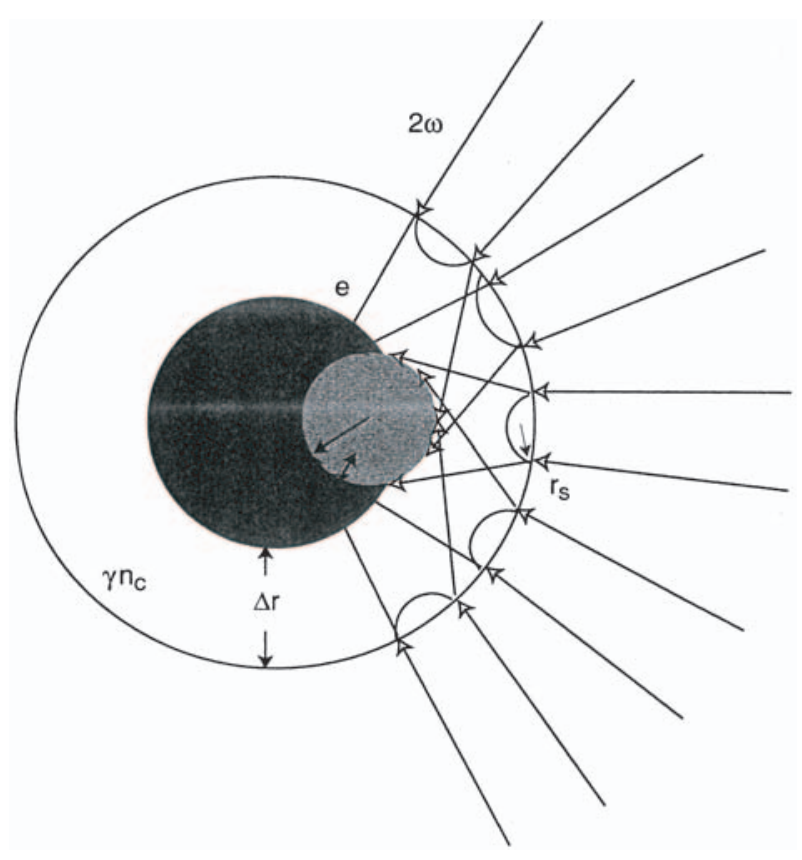

Fig. 12. Multiple ignitor beams aimed to a common hot spot.

rate still remains fast enough to give way to the disastrous nonlinear filamented behavior. To clear up those tantalizing issues, much more reliable PIC simulations are badly needed. New impressive CPU facilities are presently under way to meet these challenges. In particular, they should allow to work with a large number of quasi-particles/cell and widely spaced boundaries. Moreover, other very recent simulations by Ruhl et al. [45], seem to dwarf the importance of WEI. Other singularities, such as cavitons and solitons, compatible with ponderomotive acceleration could well be monitoring the complexities of the REB plasma in FIS conditions.

This highly controversial situation has then led some authors (Logan [46] for instance) to propose to divide initial REB density into numerous smaller ones pertaining to a number of converging subbeams (Fig. 12), toward hot spot location. Then, separated laser spots can launch hot electrons independently while collective magnetic forces may help focus multiple electron beams to the hot spot.

An intrigning and related proposal due to Malkin and Fish [47] advocates the controlled taming of a fully developed Langmuirlike turbulence underlying the cavitonsoliton nonlinearities. Then, one could expect stabilizing those collective modes through the strong density gradient in the outer layers while using them when fully developed in the density flat inner core to induce a well localized REB stopping therein.

\section{Wedged (cone-angle) REB propagation}

Although 3D simulations are not yet providing us with a convincing propagation pictures, experiment is hopefully presently providing inspiring guidance.

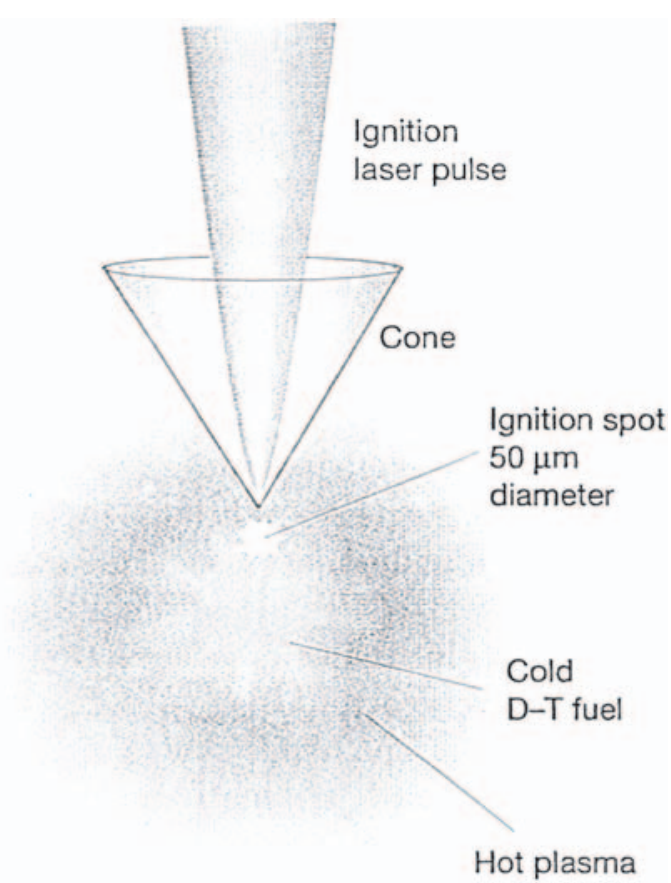

Fig. 13. The fast ignition concept. A roughly spherical mass of compressed deuterium-tritium (D-T) plasma is formed by the implosion of a hollow spherical shell of D-T around a hollow metal cone. A petawatt laser focused at the apex of the cone creates an intense beam of electrons moving at almost light speed, which ignites the D-T fuel. Kodama et al. tested, this idea for the first time in a scaled-down sub-ignition experiment using a deuterated polymer to simulate the D-T fuel pellet. Their experiment uses a short 60 -joule laser pulse (0.06 petawatt, 1 picosecond duration) to increase the number of fusion events tenfold relative to the same experiment without the 60 -joule pulse [48].

Up to now, most of REB-target experiments have been only REB interaction with solid matter or cold gas.

The very recent experiment by Kodama et al. [48] is the first to combine production of compressed matter in a laser-driven implosion with picosecond heating by a laser pulse timed to coincide with peak compression. This is a small-scale test of the fast-ignition concept and uses a small spherical shell of deuterated polystyrene to simulate the D-T ice. Because the target has no tritium, it produces D-D fusion instead of D-T fusion. The shell is formed around a hollow gold cone to provide an access path for the ignitor beam to the fuel, which is compressed to a diameter of $40 \mu \mathrm{m}$ at $50 \mathrm{~g} \mathrm{~cm}^{-3}$ (Fig. 13) [49].

Their first results are encouraging. The thermonuclear yield of neutrons from D-D fusion is increased tenfold by irradiation at peak compression with a short 60 joule laser pulse. This means that $1 \%$ of the temperature rise needed for ignition was achieved using only $0.1 \%$ of the theoretically required ignitor beam energy. They also note that the longer, 1200-joule laser pulse that drives fuel compression has to be increased to 2800 joule to achieve the same increase in neutron yield without the 60joule short-pulse heating highlighting the efficiency of the 
fast-ignition approach. Preliminary analysis suggests greater than $20 \%$ energy transfer efficiency from the short pulse laser to heating of the compressed cone, which is adequate for full-scale fast ignition.

This heating efficiency has been confirmed in the mean time by experiments using 1-petawatt, 500-joule ignition pulses [48], so the longer-term prospects for full-scale fast ignition look good. Stepping up to megajoule laser drivers, it might even lead to a 300-fold energy gain, and could initiate serious efforts worldwide to produce fusion energy by fast ignition. However, experience with other fusion energy projects should temper our enthusiasm for fast ignition. At such an early stage, this new approach to fusion energy should be still viewed as promising, but speculative, until much more work has been done. In the future a due attention would have to be given to any spurious mixing zone involving the cone material with the compressed DT. Apparently, the lateral cone does not spoil the implosion symmetry.

\section{Hot spot production}

Now, we stress the final stage of REB propagation in the compressed core. We assume that the e-beam has been able to make its way up to the highest density zone in Figure 10. So, we model the REB propagation in a cylindrical geometry.

The REB interaction physics contemplated here looks like a remake of a very similar one envisionned in the midseventies [50] for a REB direct driven compression. However, the present targets are at least five hundred times more dense than the former ones. In order to coordinate their proposal, Tabak et al. [8] advocate a $6 \mu \mathrm{m}$ range for $1 \mathrm{MeV}$ electrons fully stopped in $300 \mathrm{~g} / \mathrm{cm}^{3}$ DT fuel. Such a figure looks reasonable for igniting a hot spot much smaller than the surrounding dense compressed DT core. The REB energy range considered is fixed by the laser irradiance through the relationship $\left(\lambda_{\mu}=1 \mu \mathrm{m}\right)[51]$

$$
T(\mathrm{MeV})=0.511\left\{\left[1+0.7\left(\frac{I}{I_{18}}\right) \lambda_{\mu}^{2}\right]^{1 / 2}-1\right\}
$$

with $1 \leq I / I_{18} \leq 20$ so $0.152 \leq T(\mathrm{MeV}) \leq 1.44$.

Initial FIS prescriptions [8] recommend a $3 \mathrm{~kJ}$ REB energy at $1 \mathrm{MeV}$ for hot spot ignition. This amounts to a current $\sim 3 \times 10^{8}$ Amp. Considering a compressed core radius $\sigma \cong 50 \mu \mathrm{m}$ a plausible channel radius $a=\sigma / 4$ yields an average beam density $n_{b} \sim 1.3 \times 10^{22} \mathrm{e}-\mathrm{cm}^{-3}$. It should also be noticed that a $300 \mathrm{~g} / \mathrm{cc}$ DT core at the usual keV temperature [14] appears as a fully ionized and weakly coupled $\left(\Lambda \sim 5 \times 10^{-3}\right)$ hydrogenic plasma with

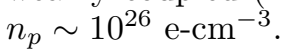

This huge current is expected to pinch the REB through an azimutal magnetic field $B_{0} \sim 4.8 \times$ $10^{10} \mathrm{G}$. Nonetheless, the linear beam density is still able to secure a very high space charge with Budker parameter $\frac{n_{b} e^{2}}{m_{e} V_{b}^{2}} \geq 10^{3}$, so the standard Alfven-Lawson limit is easily overcome. Such enormous $B_{0}$ values could a priori be a concern for the REB-target interaction itself.

Fortunately, the resulting target electron Larmor radius $\sim 3.8 \times 10^{-8} \mathrm{~cm}$ still remains much larger than the corresponding Debye length $\sim 5 \times 10^{-9} \mathrm{~cm}$ and the mean particle interdistances $\sim 1.35 \times 10^{-9} \mathrm{~cm}$ in a DT compressed at $300 \mathrm{~g} / \mathrm{cc}$ and $5 \mathrm{keV}$ temperature.

As a consequence, it is the target density which takes responsibility for shaping the energy loss and multiple scattering processes which we consider now.

The ratio $n_{b} / n_{p} \leq 10^{-4}$ demonstrates that in spite of its huge current the REB should be taken dilute in the overcompressed target with a mean electron interdistance larger by a good order of magnitude compared to that in the target. The REB-target interaction is then reducible to that of a linear superposition of isolated charges. Focusing attention on the most significant stopping mechanisms, we include binary electron-electron collisions through a plasma-adapted Møller expression [52]

$$
\begin{aligned}
-\frac{d E}{d x}=\frac{2 \pi n_{p} e^{4}}{m_{e} \beta^{2} c^{2}} \times[ & {\left[\ln \frac{1}{2 \tau_{\min }}+0.125\left(\frac{\tau}{\tau+1}\right)^{2}\right.} \\
& \left.-\frac{(2 \tau+1)}{(\tau+1)^{2}} \ln 2+1-\ln 2\right]
\end{aligned}
$$

with $\tau_{\min }=$ ratio of projectile electron wavelength $\lambda_{e}$ to target Debye length $\lambda_{D}, \tau=\gamma-1$ in terms of the usual Lorentz parameters $\beta=V / c, \gamma=\left(1-\beta^{2}\right)^{-1 / 2}$, and the excitation of Langmuir collective modes [53]

$$
-\frac{d E}{d x}=\frac{2 \pi n_{p} e^{4}}{m_{e} \beta^{2} c^{2}} \ln \left[\frac{V}{\omega_{p} \lambda_{D}} \times\left(\frac{2}{3}\right)^{1 / 2}\right]^{2}
$$

in terms of the target electron plasma frequency $\omega_{p}$.

We are entitled to restrict to a continuous slowing down approximation because large and sudden energy losses are likely to happen very rarely. This point is well documented by the fact that a bremsstrahlung contribution comparable to the above ones would request a beam energy $\sim 800 /(Z+1) \mathrm{MeV}$ much larger $(Z=1)$ than those considered here. Also, electron-position pair production remains totally negligible below $10 \mathrm{MeV}$. However, in contradistinction to the simpler ion stopping [54] case we have to give up the straight line approximation for the projectile trajectory. Due attention has now to be paid to the quasielastic and highly erratic motion of the relativistic electrons experiencing multiples scattering on target ions. Such a process is essentially quantified by the square average deflection per unit path length $(Z=1, A=2)$ [55].

Putting together the stopping contributions (2) and (3) allows us to compute a continuous range for a 90 per cent energy loss of $1 \mathrm{MeV}$ electrons, as $R=$ $35.66 \mu \mathrm{m}$ for a stopping target with $300 \mathrm{~g} / \mathrm{cc}$ and $5 \mathrm{keV}$. It should be noticed that the range in a hot target with classical electrons is shorter than the corresponding one at $T=0$ with fully degenerate ones. The latter being notoriously less responsive to the projectile electric field. 
This $R$ value is comparable with the core extension $\sigma$. So, we really need an efficient packing mechanism to wind the projectile trajectories within a much smaller domain in the compressed core. This winding process is easily quantified by the maximum penetration depth $l_{0}$ of the given REB.

The precise calculation of this parameter has been for many years the hard core of that subfield of nuclear and particle physics devoted to particle detection. In the present context, we find it very useful to use a classical result due to Hemmer and Farquahr [56] which is based on stochastic arguments summarizing a great deal of previous efforts. So, considering a slab thickness containing every projectile trajectory whatever their orientation with respect to beam axis is, one gets the simple relationship

$$
R=l_{0}+\frac{1}{2} \frac{l_{0}^{2}}{\lambda}+\frac{1}{2} \frac{l_{0}^{3}}{\lambda^{2}}
$$

between the continuous winded range $R$ and the maximum penetration depth $l_{0}$.

Figure 14a features simultaneously $R$ and $l_{0}$ for the energy range $0.5 \leq T(\mathrm{MeV}) \leq 15$. In order to qualify the FIS as a coherent ignition scenario, we have also to request a sufficiently short stopping time which allows for an adequate hot spot extension highlighted by $4 \leq l_{0} \leq$ $18(\mu \mathrm{m})$.

The corresponding $t_{\text {stop }} \sim 10^{-13}$ s exhibited in Figure 14b seems compatible with further equilibration time between hot electrons and thermonuclear ions in ignited target.

Those results are indeed putting the interaction physics of the FIS on serious grounds. However, other related issues such as the conversion efficiency of the laser light into energetic REB have also to be positively addressed. Such concerns are motivating some authors [57] to advocate an even more precompressed DT core. This explains that we investigate in Figure 15, the target density dependence of the above results for a $1 \mathrm{MeV}$ REB and a target density between $300 \mathrm{~g} / \mathrm{cc}$ and $1000 \mathrm{~g} / \mathrm{cc}$. Then, we really witness a drastic reduction of $R, l_{0}$ and $t_{\text {stop. }}$.

On the other hand, we also proceeded to a systematic variation of the target temperature between 1 and $5 \mathrm{MeV}$. The above results are then left practically unchanged. Large $T$ variations remain below $2.5 \%$.

The above relativistic stopping expressions (2-3) refer to the interaction of a single electron projectile with the target plasma. However, in view of the very large e-beam density, the occurrence of dynamical inflight correlations between electron projectiles should also be accounted for. Recent investigations [58] demonstrate that even for projectile interdistances $\sim n \lambda_{D}$ with $n \geq 10$ and $\lambda_{D}$, target plasma Debye length, such an effect yields to a significantly magnified energy loss for the e-beam. So, the local energy deposit concept appears well-grounded and the actual REB range is likely to be shorter that the above slab estimated $l_{0}$.
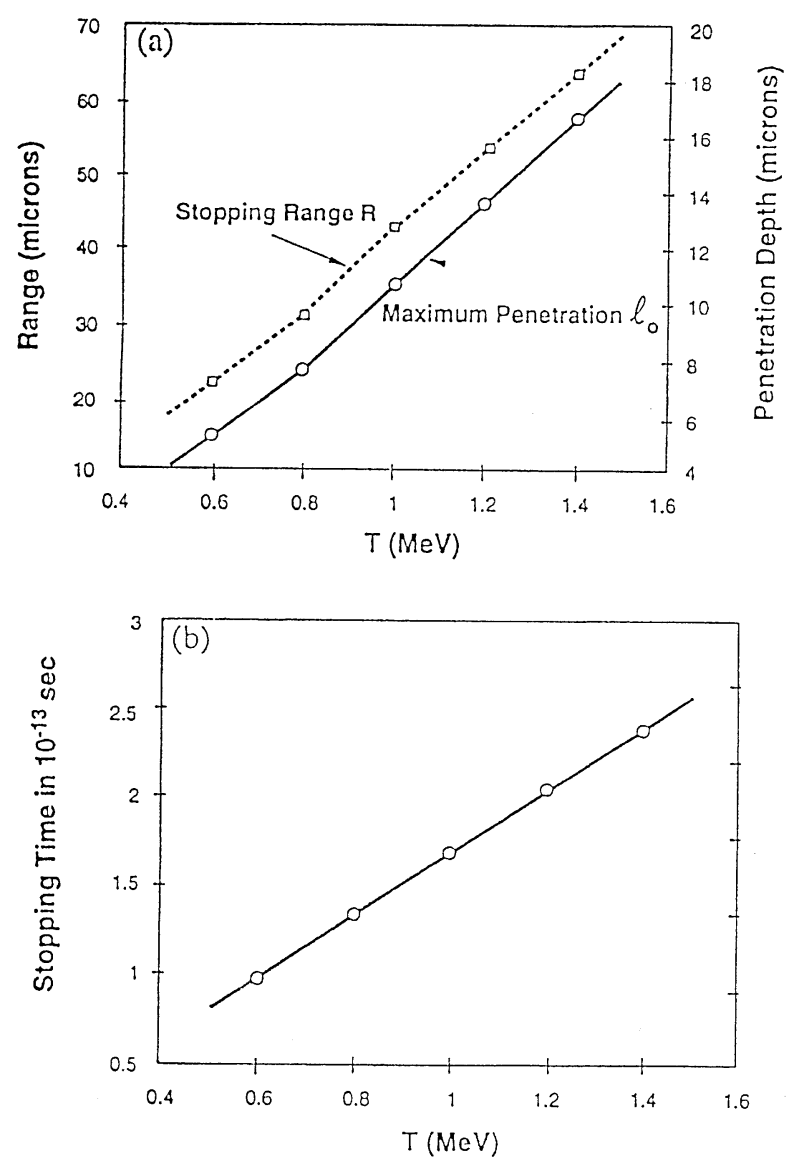

Fig. 14. (a) REB range $R(\mu \mathrm{m})$ and maximum penetration depth $l_{0}(\mathrm{~cm})$ in a $300 \mathrm{~g} / \mathrm{cc}$ DT target at $5 \mathrm{keV}$ and $0.5 \leq T(\mathrm{MeV}) \leq 1.5$; (b) corresponding stopping time $t_{\text {stop }}$ [14].

\section{Fast ignition}

Keeping in mind the above REB range estimates for complete stopping at highest density of supercompressed D-T, it could be appropriate for further elaboration of the ignition REB parameters, to simplify the previous complex pictures (Figs. 14-15) with the cylindrical-spherical interaction given in Figure 16.

A precompressed DT fuel, initially at uniform density $\rho$ (and free to expand into the surrounding vacuum), is hit by a beam of particles with assigned range $\mathcal{R}$ (a free parameter in the simulations), uniform stopping power and straight path, delivering energy to the plasma electrons (see Fig. 16). The beam is parallel and cylindrically symmetric, with uniform intensity $I_{p}$, for $r \leq r_{b}$, constant power $W_{p}$, and pulse duration $t_{p}$. (Here $\bar{r}$ is the radial coordinate.)

Here we have a spherical fuel assembly, with initial radius $r_{s}$, such that $\rho r_{s}=3.6 \mathrm{~g} / \mathrm{cm}^{2}$. One can however check that the results are nearly independent on $r_{s}$ as far as the particle penetration depth and the beam radius are much smaller than the sphere radius. When considering particles with penetration depth $R>1.2 \mathrm{~g} / \mathrm{cm}^{2}$, one has better 

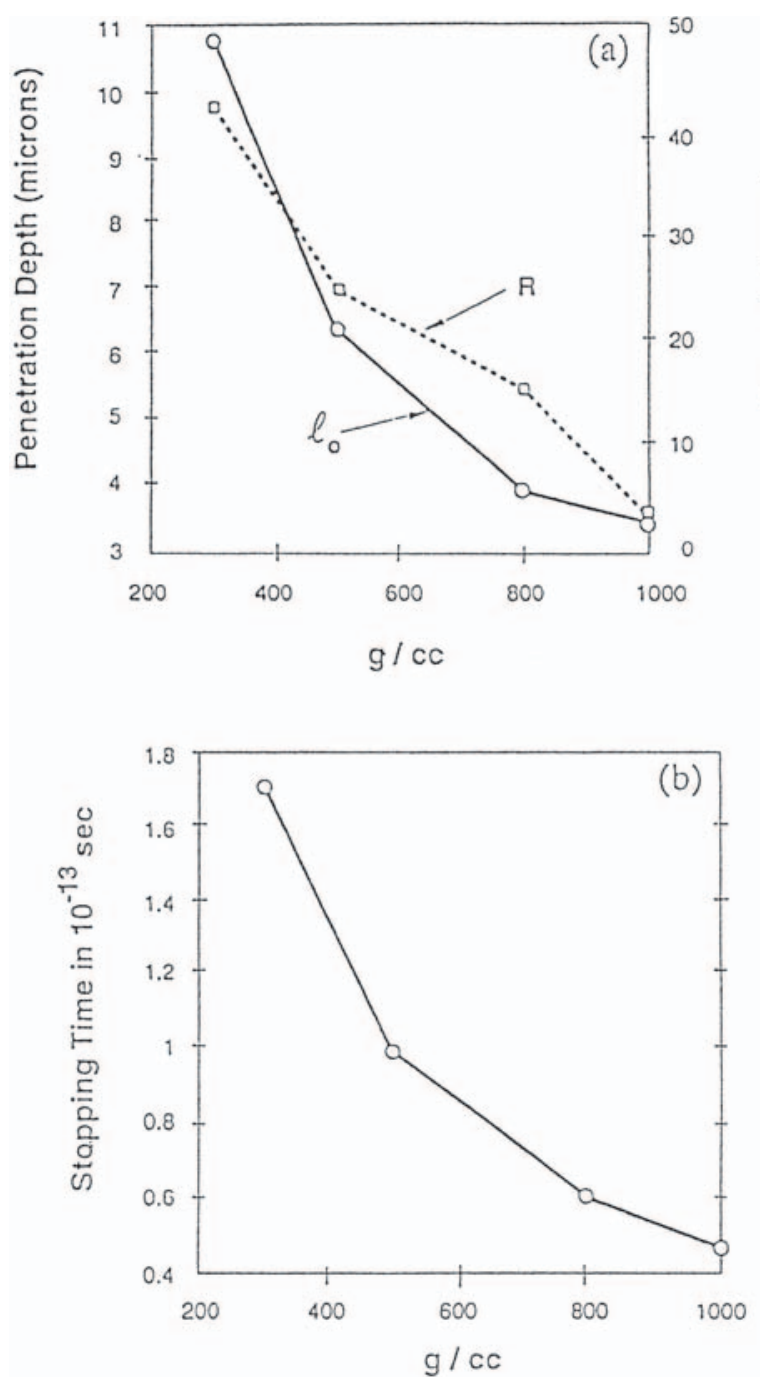

Fig. 15. (a) $R$ and $l_{0}$ for $T=1 \mathrm{MeV}$, and target density ranging from 300 up to $1000 \mathrm{~g} / \mathrm{cc}$, with $5 \mathrm{keV}$ temperature; (b) corresponding stopping time $t_{\text {stop }}$ (Deutsch et al. [14]).

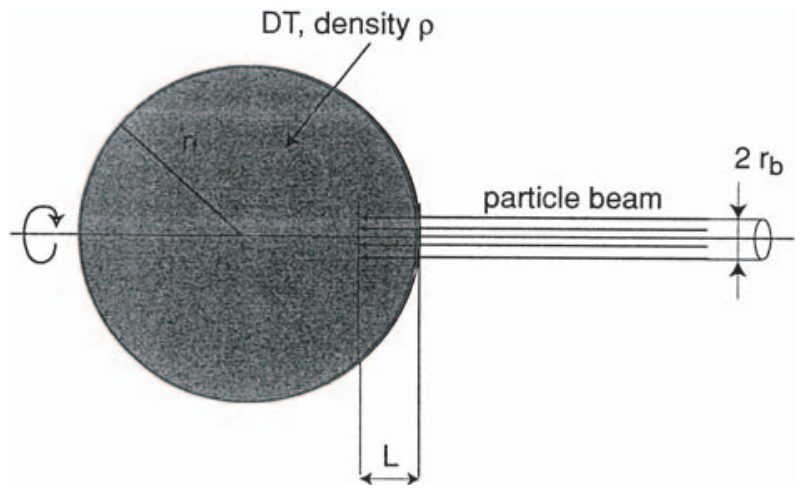

Fig. 16. Initial conditions for the model problem studied by 2D simulations: cylindrical symmetry around the horizonral axis is assumed. The radius of the compressed DT sphere always satisfies, $r_{s} \gg r_{b}$, and $r_{s} \gg L$, where $L=R / \rho$ is the particle peneration depth, and $\rho$ is the initial fuel density [59]. to considere a cylindrical fuel assembly, with radius $r_{c}$ and length $L_{c}$, such that $\rho r_{c}=3 \mathrm{~g} / \mathrm{cm}^{2}$, and $\rho L_{c}=7.5 \mathrm{~g} / \mathrm{cm}^{2}$.

The present model is a rough schematization of the interaction of the actual particle beam with the dense plasma: effects such as deflections, straggling, and Bragg peaks are neglected. While important for detailed target design, the above features are not expected to affect the results of the present investigation, where only integral features of the deposition process, and the influence of the range are of interest.

Atzeni [59] followed this approach and after an extensive parameters study based on a $2 \mathrm{D}$ radiationhydrodynamics code (DUED) he found that ignition occurs if the deposited beam energy, power and intensity simultaneously exceed thresholds values, $E_{i g}, W_{i g}$, and $I_{i g}$, respectively, which are are fitted by

$$
\begin{aligned}
E_{i g} & =140(\hat{\rho})^{-1.85} \mathrm{~kJ} \\
W_{i g} & =2.6 \times 10^{15}(\hat{\rho})^{-1} \mathrm{~W} \\
I_{i g} & =2.4 \times 10^{19}(\hat{\rho})^{-0.95} \mathrm{~W} / \mathrm{cm}^{2}
\end{aligned}
$$

with $\hat{\rho}=\rho /\left(100 \mathrm{~g} / \mathrm{cm}^{3}\right)$.

Those values are considerably larger than those initially proposed by Tabak et al. [8]. At density $\rho=$ $300 \mathrm{~g} / \mathrm{cc}$, one has $E_{i g}=18 \mathrm{~kJ}$ in equation (5) vs. $E_{i g}=$ $3 \mathrm{~kJ}$ given previously.

Atzeni [59] also determined the energy, power and intensity thresholds for ignition of a fuel at a density $\rho=300 \mathrm{~g} / \mathrm{cm}^{3}$, as a function of the penetration depth of the heating particles, of the radius of the beam (and hence of the heated spot), and of the pulse duration. Detailed results referring to the values of the range $\mathcal{R}=0.3$, $0.6,1.2 \mathrm{~g} / \mathrm{cm}^{2}$ are illustrated in Figures 17 for $E_{i g}$.

The following trends are apparent:

(1) For a given value of the range, the key pulse parameter is energy for short pulses and relatively small focal spots, it is power for long pulses and small spots and it is flux for large spots and long pulses. The characteristics length (spot radius, $r_{c h}$ ), discriminating the above regimes is well approximated by $\rho r_{c h}=0.6 \mathrm{~g} / \mathrm{cm}^{2}$, and is independent of the particle range. The analogous characteristic time, $t_{c h}$, instead, is a growing function of the range, being $t_{c h} \cong 20 \mathrm{ps}$ for $\mathcal{R}=0.30 \mathrm{~g} / \mathrm{cm}^{2}$, and $t_{c h} \cong 40 \mathrm{ps}$ for $\mathcal{R}=1.2 \mathrm{~g} / \mathrm{cm}^{2}$.

(2) The minimum value of the ignition energy is about $13.5 \mathrm{~kJ}$ (and is achieved for range $\mathcal{R} \leq 0.6 \mathrm{~g} / \mathrm{cm}^{2}$, $\rho r_{b} \cong 0.3 \mathrm{~g} / \mathrm{cm}^{2}$ and $\left.t_{p} \cong 10 \mathrm{ps}\right)$, but corresponds to rather high power $\left(W_{p} \cong 1.5 \times 10^{15} \mathrm{~W}\right)$ and intensity; ignition with $18-20 \mathrm{~kJ}$ is instead achieved in a relatively large portion of the parameter space, and for a wide interval of values of the range $\left(t_{p} \leq 20 \mathrm{ps}\right.$; $\left.r_{b} \leq 20 \mu \mathrm{m} ; \mathcal{R} \leq 1.2 \mathrm{~g} / \mathrm{cm}^{2}\right)$ and with considerably smaller beam power (see below). Even for $R=2 \mathrm{~g} / \mathrm{cm}^{2}$ ignition can be achieved with $20 \mathrm{~kJ}$ of delivered beam energy.

(3) The beam power required for ignition, $W_{i g}$ is, for a given radius, a decreasing function of the pulse length, approaching a constant value for long pulses. For a given pulse length, $W_{i g}$ is nearly independent on the 
a)

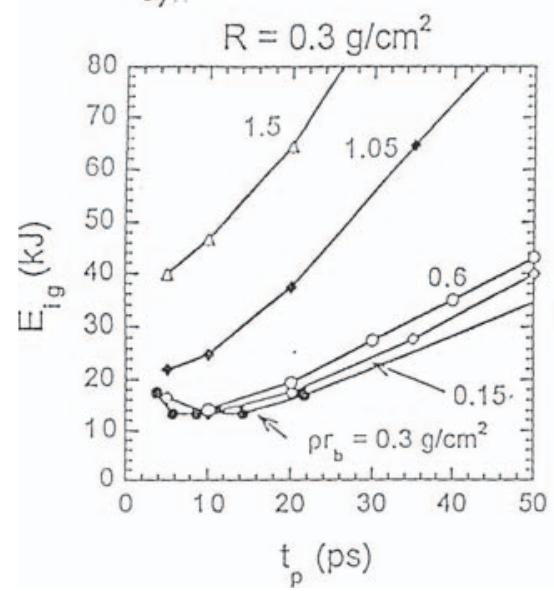

b)

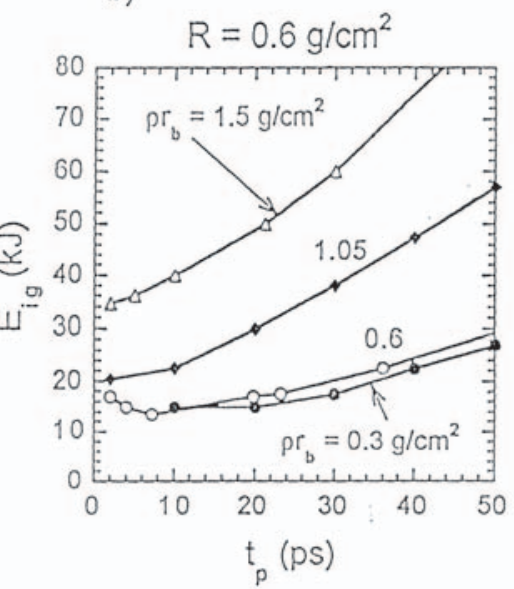

c)

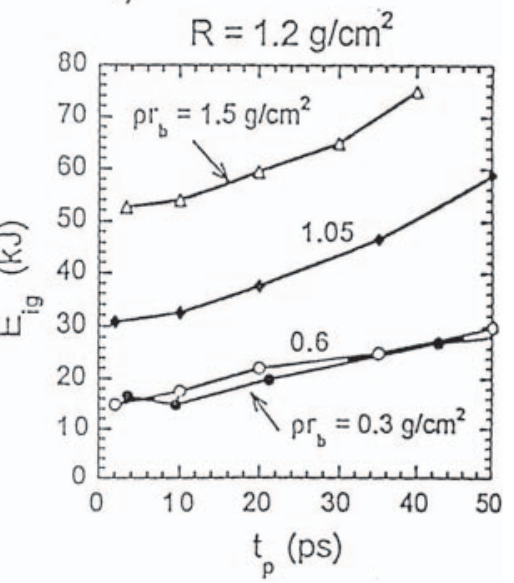

Fig. 17. Minimum pulse energy for ignition, vs pulse duration, for DT fuel at $\rho=300 \mathrm{~g} / \mathrm{cm}^{3}$ and different values of the beam radius $r_{B}$ (see labels on the curves) and of the penetration depth $R$ of the fast particles; (a) $R=0.3 \mathrm{~g} / \mathrm{cm}^{2} ;(\mathrm{b}) R=0.6 \mathrm{~g} / \mathrm{cm}^{2}$; (c) $R=1.2 \mathrm{~g} / \mathrm{cm}^{2}$. Results for $R=0.15 \mathrm{~g} / \mathrm{cm}^{2}$ (not shown) and small focal spot are close to those for $R=0.3 \mathrm{~g} / \mathrm{cm}^{2}[59]$.

beam radius for $\rho r_{b} \leq 0.6 \mathrm{~g} / \mathrm{cm}^{2}$, and is a growing function of $r_{b}$ at larger radius.

(4) The beam intensity for ignition, $I_{i g}$ varies with the duration of the pulse in a manner analogous to the power, but has an opposite dependence with the spot size, being larger for smaller radius, and then saturating, approaching a minimum value for $\rho r_{b} \geq 1 \mathrm{~g} / \mathrm{cm}^{2}$.

(5) A most important feature, is the really weak dependence of the ignition energy (and of the other pulse parameters) on the particle range, in particular for relatively short pulses and relatively small focal spot.

\section{Production of $\mathrm{MeV}$ protons}

One of the most exciting results recently obtained in this area of research is the observation of very energetic beams of protons, generated during the interaction of ultraintense short pulses with solid targets. In a number of experiments, performed with different laser systems and in different interaction conditions, protons with energies up to several tens of $\mathrm{MeV}$ have been detected behind thin foils irradiated with high-intensity pulses (Clark et al. [60]; Maksinchuk et al. [61]; Snavely et al. [62]). In these experiments, it was seen that the particle beams are directed along the normal to the back surface of the target, and have a small angular aperture at the highest energies. As proton beams are observed even using targets which nominally do not contain hydrogen, protons are thought to originate from hydrocarbon impurities located on the target surfaces or from bulk contamination of the target. There has been a strong debate whether the source of the energetic protons is located at the front or at the back surface of the solid target. Arguments bases on the angular distributions of the proton energy across the beam have been used by Clark et al. [60] to support the hypothesis of a proton source located at the front surface of the target. On the other hand, the fact that the proton beam is perpendicular to the target back surface (rather than collinear with the interaction beam) supports the hypothesis that the proton acceleration takes place at the back of the target (Snavely [62]). In this case, the protons would be accelerated by the enormous electric field (approximately megavolts/micron) set up by the fast electrons leaving the target (Hatchett et al. [63], Wilks et al. [64]).

The particular properties of these beams (small source size, high degree of collimation, short duration, energy dependence on the target characteristics) make them of particular interest in view of possible applications. Among these, their use as the ignition trigger in the fast ignitor scheme (Roth et al. [65]) and as a probe in high-density matter investigations (Borghesi et al. [66]) have been proposed.

The protons were produced by focusing the CPA pulse onto $25 \mu \mathrm{m}$-thick $\mathrm{Al}$ foils, and detected using layers of radiochromic film (RCF; McLaughlin et al. [67] and plastic tracks detectors (Enge, [68]) placed in a stack at the back of the target, typically at a distance of about $2 \mathrm{~cm}$. It was found that the proton beams have high brightness, typically with $10^{12}$ protons with energy above $3 \mathrm{MeV}$ per shot (for laser irradiance of the order of $5 \times 10^{19} \mathrm{~W} \mathrm{~cm}^{-2}$ ). As observed in previous experiments, the beams were highly directional, propagating along the normal to the back surface of the target with small angular divergence (about $15^{\circ}$ for $10 \mathrm{MeV}$ protons).

The energy deposited within each film layer can be extracted from the absolutely calibrated film. By doing this for each film layer and fitting an exponential energy dependence, an estimate of the proton energy spectrum and total energy can be extracted from the film data.

During the experiments, the source size of the proton beam was estimated using a penumbral edge method, setting an upper limit of $15-20 \mu \mathrm{m}$ diameter for the source of $10 \mathrm{MeV}$ protons. It is presently unclear whether the observed source is real or rather a virtual one (with the protons emitted from a larger area with finite angular divergence and spread). In any case, the measured source 
size is small enough to permit the implementation of point projection imaging schemes, and sets the spatial resolution of imaging applications.

A test was carried out to investigate the proton acceleration mechanism, and in particular its dependence on the scale length of the plasma at the back of the target (Mackinnon et al. [69]). This was done to confirm the assumption that the protons are electrostatically accelerated at the back surface of the target. To create a finite plasma scale length, a preformed plasma was created by focusing a 600 -ps-duration laser pulse at $\lambda=0.527 \mu \mathrm{m}$ onto the back surface of the foil with an $f / 10$ lens. The heating pulse energy, focal spot, and relative timing were all controlled on a shot-to-shot basis. For most shots, the energy on target was 10-J within a foacal spot of $300 \mu \mathrm{m}$ (FWHM), and the heating pulse started 250 ps before the interaction pulse, thus giving a mean intensity on target of $3 \times 10^{13} \mathrm{~W} \mathrm{~cm}^{-2}$. As a matter of fact, the proton production was very different when the heating pulse was incident on the interaction pulse, and formed a plasma with a scale length at critical of the order of $20-50 \mu \mathrm{m}$.

Confirmation that the proton signal was very much reduced in the preformed plasma case was obtained by etching one of the three Mylar laryers between the first two RC film layers. There was a one to one correspondence between the track features in the etched Mylar and the $\mathrm{RC}$ data. For the unperturbed case, in each layer the track density within the proton beam was very high and the signal fell off very sharply at the edge region of the spot. In contrast, for the preformed plasma case, the track density is high only on the first Mylar layer and it drops off very rapidly to background levels before the second layer of $\mathrm{RC}$ film. From this we can infer that the energy cut-off is actually lower than 5-6 MeV. The same behavior was observed in recent measurements when much thicker targets (up to $250 \mu \mathrm{m}$ thick) were used. Even in this case the protons beam disappeared completely when a small plasma (with scale length of a few tens of microns) was produced on the back of the target, as predicted by electrostatic acceleration in the Debye sheath at the back surface of ther target (Hatchett et al. [63], Wilks et al. [64]).

It should be noticed that rear protons are likely to emerge with a comoving electron beam and with a very small emittance $0.006 \pi \mathrm{mm} . \mathrm{mrad}$, i.e. a hundred time smaller than the usual linac one.

\section{Fast ignition with protons}

Many experimenters [60-63] have very recently confirmed that energetic protons $(5-70 \mathrm{MeV})$ are dragged away from a rear surface of ultra-intense laser illuminated plastic foils. The concept of proton driven FIS [65] thus offers a way for circumventing the difficulties of ion acceleration, pulse compression, focusing, and transport. Moreover, it can be applied to both direct drive and indirect drive ICF geometries.

This concept for fast ignition using laser-accelerated protons is depicted in Figure 18. Multiple petawatt-class laser beams are focused onto a target which is attached to the hohlraum in an indirect drive ICF geometry. An intense beam of protons is accelerated from the rear, nonirradiated surface of the target, which is spherical in shape in order to ballistically focus these protons to a small spot.

In Figure 18 precompression is secured with the standard HIF scenario using two lateral converters. The given hohlraum might be as well triggered by lasers or Z-pinches.

A thin metal window in the hohlraum wall protects the rear surface of the proton target from preheat caused by the intense solf $\mathrm{X}$-ray radiation which is generated in the hohlraum by the implosion driver. A vacuum gap between the proton target and the protective window of $\sim 200 \mu \mathrm{m}$ could be required to provide a sufficient distance for the proton acceleration and a buffer against the hydrodynamic expansion of the hohlraum entry window due to the heat deposited in it.

Extremely high proton currents can be focused ballistically into the hot spot without space-charge driven defocusing, because the protons are accelerated as a plasma with an accompanying electron cloud which provides space-charge neutralization. Once the protons penetrate the thin metal window, we assume that their space charge will be neutralized by the plasma within the hohlraum, confirmation of which requires further beam transport calculations. However, since the proton beam density is everywhere much lower than the hohlraum plasma density, this assumption appears reasonable and we consider proton beam defocusing effects from multiple scattering and emittance only.

The optimum proton beam size is set equal to the range in the compressed fuel, and the maximum proton pulse duration is determined by the expansion time of the hot spot, given by the hot spot size divided by the sound speed, $r_{0} / c_{s}$.

Laser produced protons have a range appropriate to create a hot spot in deuterium-tritium (DT) fuel, can be transported for a distance of a few $\mathrm{mm}$ and focused to spots of $15 \mu \mathrm{m}$ radius. The scheme they suggested then consists in a standard ICF hohlraum, where a capsule containing DT fuel is ablatively compressed by thermal radiation, and a properly shaped proton source placed just outside the hohlraum (see Fig. 18). At a time close to maximum compression of the fusion fuel, a bundle of ultra-intense laser beams is focused on this second target, which emits a burst of protons, directed towards the compressed fuel. Using standard range computations and the results of reference [59] concerning particle beam requirements for fast ignition, Roth et al. estimated that 7-10 kJ of $15-23 \mathrm{MeV}$ protons, reaching the fuel in a $15-20 \mathrm{ps}$ pulse and focused onto a spot of $15 \mu \mathrm{m}$, are required to fast ignite the fuel compressed to a density of $400 \mathrm{~g} / \mathrm{cm}^{3}$. (Energy, radius, and pulse duration scale with density as $E_{18} \propto \rho^{-1.85} ; r_{b} \propto \rho^{-1} ; \tau_{p} \propto \rho^{-0.85}$, respectively).

However, these results refer to an ideal particle beam (with range just fitted to the hot spot size and independent on temperature and density) interacting with a homogenous fuel sphere. In fact since protons are produced with a wide-energy spectrum $[60,62,63]$, pulse power and length 


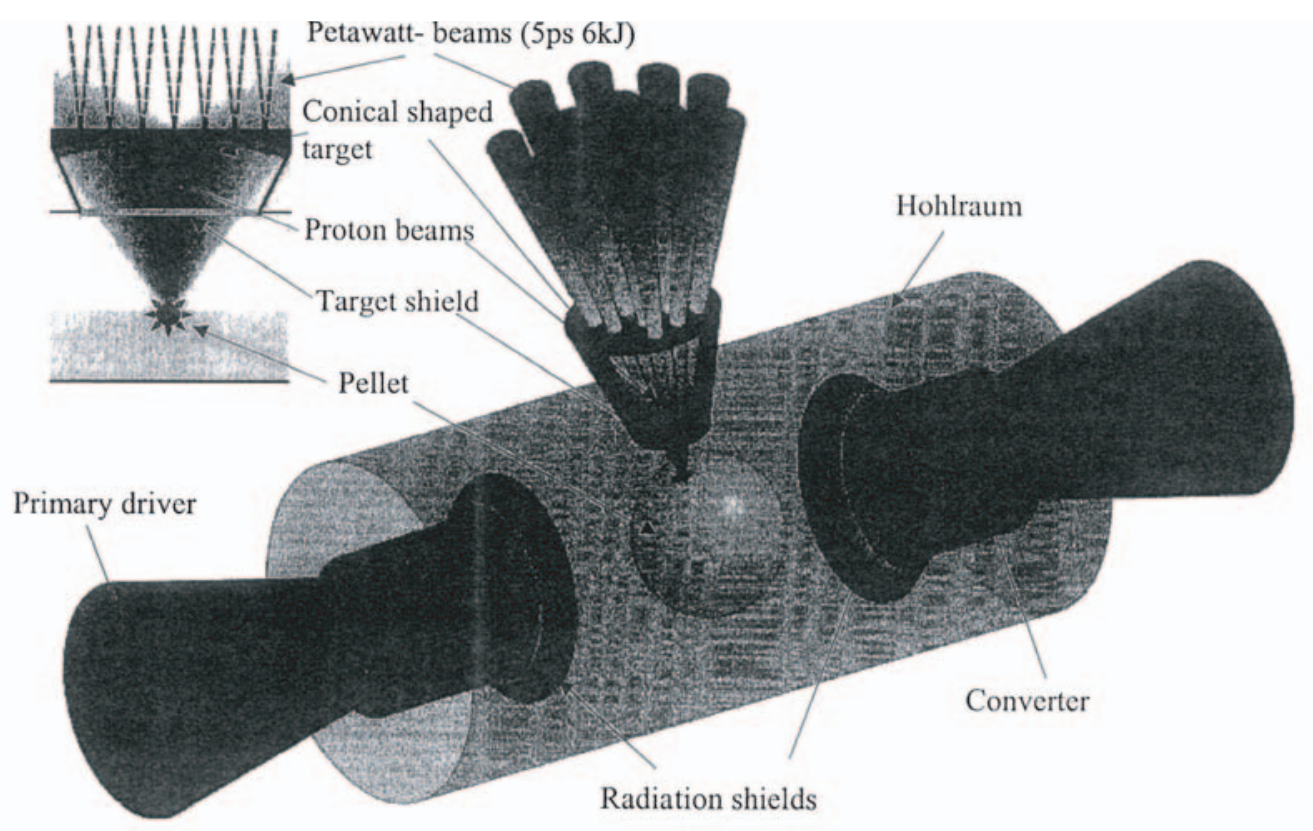

\section{Eㅗㅛㅛㅛ Phasma}

Fig. 18. Indirectly driven fast ignition using a laser accelerated proton beam (not to scale). The rear surface of the laser target is shaped to focus the ion beam into the spark volume (Roth et al. [65]).

will be a function of both spectrum and distance between source and compressed fuel.

Proton range is a function of both proton energy and plasma conditions. In addition, the compressed fuel will not be isochoric. A more complete analysis is therefore needed. A first step in this direction was taken up by Atzeni et al. [70].

They have showed that due to the range dependence on plasma temperature and power spread due to proton velocity dispersion, the minimum ignition energy (for a homogeneous fuel sphere) is larger than that estimated in reference [65]. A good fit to results referring to a simple proton energy distribution is $E_{i g}=$ $90\left[d(\mathrm{~mm})^{0.7} /\left[\rho / 100\left(\mathrm{~g} / \mathrm{cm}^{3}\right)\right]^{1.3} \mathrm{~kJ}\right.$.

Proton generation transport and focusing on target are crucial issues for such a new scheme. In particular, the very large currents involved may require neutralized propagation, with the fast protons accompagnied by comoving electrons. One should then properly speak of a plasma beam, although the energy carriers would in any case be the fast protons.

It is indeed a fortunate experimental occurence that the $\mathrm{MeV}$ protons produced from the rear of the target foil with a smallest emittance, are also propagating with comoving electrons providing charge- and currentneutralization. The authors of reference [70] use again 2D Lagrangian simulations [59].

Their code employs a three-temperature plasma model and includes a real-matter equation-of-state, appropriate opacities, collisional transport, nuclear reactions and transport of fusion products. Proton-plasma interaction is dealt with by standard binary collision theory using a model similar to those usually employed in ion beam driven ICF studies.

In this connection, it should be noticed that proton stopping through excitation of collective plasma modes should also be properly included. Actually, several groups are presently devoting a closer scruting to this important issue.

Nonetheless, those preliminary calculations already provide a meaningful analysis for the scenario of proton driven fast ignition.

Atzeni et al. [70] thus obtain proton ranges as a function of target plasma temperature (Fig. 19).

As shown in Figure 19, the range of multi-MeV proton is nearly constant at relatively low plasma temperature, then increases substantially as the DT temperature exceeds some threshold. This is easily understood by observing that the fast protons are slowed down by the plasma thermal electrons, and that the stopping power decreases as the relative velocity of the colliding particles increases. From Figure 19 we see that at plasma temperature $T=5-$ $10 \mathrm{keV}$, protons with $\varepsilon_{p} \geq 10 \mathrm{MeV}$ have range longer than the optimal value for fast ignition.

A related stopping calculation due to Roth et al. [65] makes use of the standard stopping model (SSM) [54] which also includes those plasma modes excitation. Required conditions for hot spot ignition feature $\rho_{0} r_{0}=$ $0.6 \mathrm{~g} / \mathrm{cm}^{2}$ at $k T=10-12 \mathrm{keV}$, with a compressed fuel density of $\rho_{0}=300 \mathrm{~g} / \mathrm{cm}^{3}$. A proton ignitor beam generated was considered from a target placed $220 \mu \mathrm{m}$ outside of the hohlraum. After penetrating the wall it traverses the 


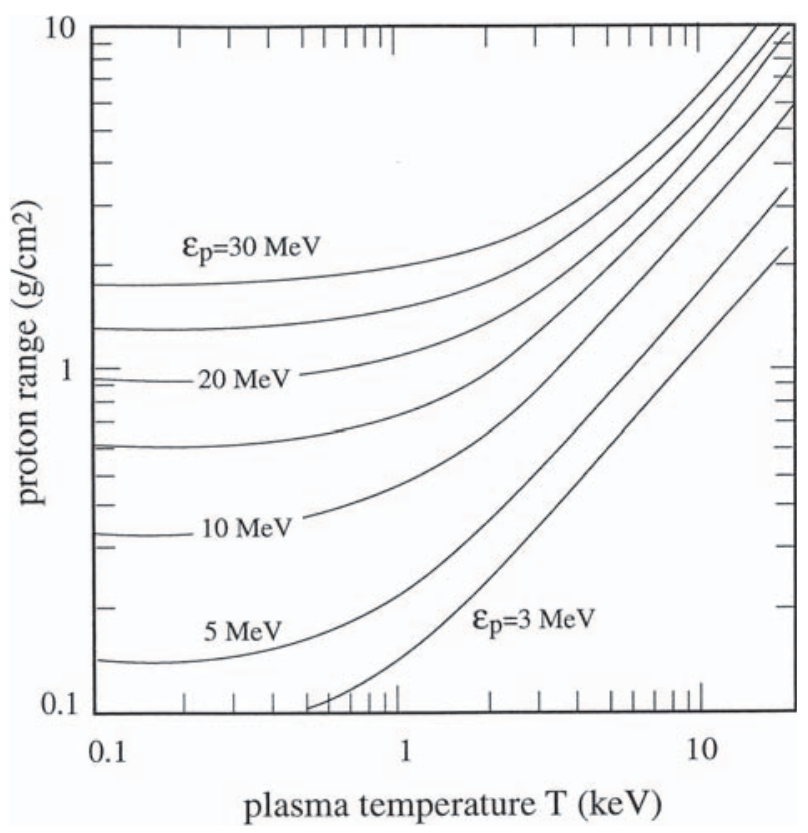

Fig. 19. Range of protons with different initial energy $\varepsilon_{p}$ in DT plasma at density $\rho=400 \mathrm{~g} / \mathrm{cm}^{3}$ versus the plasma temperature $T$ [70].

hohlraum filled with plasma originating from wall blowoff and pellet blowoff and finally impinges on the compressed fuel producing the hot spot.

For the proton transport calculation, the distance from the source to the hot spot was divided into $1 \mu \mathrm{m}$ thick layers, for each of which the material and plasma parameters were determined. The electron density distribution close to the compressed fuel was chosen to rise from $0.1 n_{c}$ $\left(n_{c}=\right.$ critical density at $\left.\lambda_{\text {laser }}=350 \mathrm{~nm}\right)$ to a fully ionized hydrogen plasma at $300 \mathrm{~g} / \mathrm{cm}^{3}$. The calculation of the energy deposition in the rest of the fuel assumed a constant density (isochorically compressed target). For each layer, the specific proton energy loss, and hence the associated energy deposition, was calculated for protons having initial energies between 5 and $35 \mathrm{MeV}$. The total energy deposited in a given layer was determined by convolving with a measured proton energy spectrum. To include the energy dependence on the focusability, the measured beam emittance for each energy was used to calculate the respective spot size and therefore the fraction of the protons in the region of interest.

Figure 20 shows the calculated energy deposition profiles for protons having initial energies between 15 and $23 \mathrm{MeV}$ (dashed curves). We find that protons within this energy range satisfy the first requirement for an ignitor beam, that they deposit the bulk of their energy (at the Bragg peak of their energy-loss profile) within the optimal $0.6 \mathrm{~g} / \mathrm{cm}^{2}$ range in the compressed fuel. The solid curve in Figure 20 shows the total energy deposition profile for protons in this optimal 15 to $23 \mathrm{MeV}$ range. The typical energy loss penetrating the $30 \mu \mathrm{m}$ gold hohlraum wall is

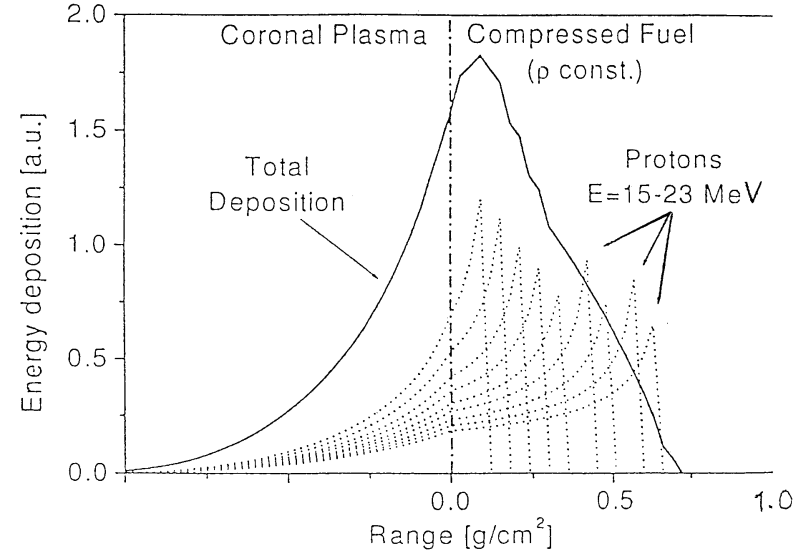

Fig. 20. Energy deposition of protons between 15 and $23 \mathrm{MeV}$ in a isochorically compressed pellet [65].

about $1 \mathrm{MeV}$, and the energy loss in the hohlraum plasma is negligible compared to the fraction lost in the coronal plasma and finally in the compressed fuel.

The second constraint on the usable range of initial proton energies is the temporal dispersion of the ignitor proton pulse, whose total duration must be short compared to the expansion time of the hot spot. For our point design fuel density, the minimum hot spot size is the $\alpha$-particle range required to sustain burn $(20 \mu \mathrm{m})$, and the maximum allowable pulse duration is $20 \mathrm{ps}$. The proton pulse duration is determined by both the intrinsic source duration and the temporal dispersion of the pulse due to the range of proton velocities and the path length from proton production target to the fuel. The acceleration mechanism provides an upper limit on the duration of the acceleration of $<10 \mathrm{ps}$, due to the cooling time of the hot electrons in the plasma sheath which drives the proton expansion. In this example, the ignitor protons are generated $220 \mu \mathrm{m}$ outsite of the hohlraum wall and we assume a NIF-type hohlraum of diameter $5.5 \mathrm{~mm}$. For protons between 15 and $23 \mathrm{MeV}$, the temporal spread of the proton pulse by velocity dispersion is $<10$ ps which, when convoluted with the intrinsic source duration, gives a total pulse duration of $<20$ ps.

The ignition energy that has to be delivered by the proton beam grows with the distance $d$ between source and dense fuel. As a mean to reduce such a distance one could use conically guided sperical targets originally proposed for fast ignition by electrons (see Sect. 4).

In this connection, Temporal et al. [70] considered recently two quite different conical guiding systems characterized by the substantially different value of the angle $\beta$ defining the spherical sector filled by the fusion target. In 
one concept $\beta<\pi / 2$. This scheme tries to merge the positive effect of convergence to the simplicity of one sidedirradiation. In addition, it allows for easy access to the compressed fuel, since fast ignitor particle beams do not have to cross the corona. In the second concept, instead, $\beta>\pi / 2$; ideally one would like to subtract from a sphere the smallest cone still allowing for delivering fast particles at the center. The main issue for both schemes is fuel compression, which requires both suitable irradiation geometry and limitation of boundary effects close to the cone surface. It appears more critical for targets with $\beta \leq \pi / 2$.

It is also assumed that conical guiding does not affect significantly implosion dynamics so that the status of the compressed fuel can be taken from the 1D simulation. This assumption is supported by the experimental results of Kodama et al. [48] and also by the simulations of Hatchett et al. [51]. In particular, Kodama et al. pointed out that the insertion of the cone reduces the compressed density by about 20\%-30\% compared with full spherical implosion.

Because of the reduced time-of-flight of the proton, the duration of the power pulse is favorably shortened from the $70 \mathrm{ps}$ [full width at half maximum (FWHM)] of the reference with $d=4 \mathrm{~mm}$ to about $8 \mathrm{ps}$ in this case. A second advantage follows from the easier access to the region of highest plasma density. The beam energy for ignition turns out to be $12-14 \mathrm{~kJ}$ for beams with $T_{p}=3-5 \mathrm{MeV}$. A word of caution is needed here, since the power pulse duration due to velocity dispersion is now comparable with the presumable duration of the proton beam emission. Thermonuclear burn of the fuel releases $246 \mathrm{MJ}$, corresponding to about $50 \%$ of the yield of the corresponding spherical capsule. This shows that the burn efficiency (fractional burn) is practically the same as for the spherical capsule. Temporal et al. [70] also consider the dependence of the burn fraction and of the target gain on the cone aperture $\beta$. Let $E_{w} / \eta_{h}$ be the energy needed to compress the whole spherical capsule and $F_{v}(\beta)=[1-\cos (\beta)] / 2$ the volume of the conical shell relative to the spherical one. If we assume that the efficiency $\eta_{h}$ is independent of $\beta$, we can write the target gain $F(\beta)$ as

$$
G(\beta)=E_{\mathrm{fus}}(\beta) /\left[F_{v}(\beta) E_{w} / \eta_{h}+E_{i g} / \eta_{F I}\right],
$$

where $E_{\mathrm{fus}}(\beta)=340 m_{D T} \phi(\beta) F v(\beta) \mathbf{M J}$ is the fusion energy, $\phi(\beta)$ is the DT fractional burn-up and $m_{D T}$ is the fuel mass of the spherical capsule in mg. The yield of the spherical capsule is $E_{\text {fus }}(\beta=\pi)=488$ MJ. Taking $E_{w}=635 \mathrm{~kJ}, E_{i g}=12 \mathrm{~kJ}$ (i.e., assuming a very small source-fuel distance), $\eta_{h}=0.3$ and $\eta_{F I}=0.12$, we estimate the maximum gain $G_{\max }=220$.

Since we expect that $\phi(\beta)$ grows with $\beta$, it follows that also $G$ should monotonically increase with the angle $\beta$ to reach its maximum value $G(\pi)$ for $\beta=\pi$.

Concerning the cases with small values of $\beta$, it is worth noting that they could allow to achieve substantial gain at smaller values of the total invested energy.

Of course, the actual viability of conically guided targets rests on the possibility of achieving the same compressions as in the spherical cases, and in limiting undesired effects associated with the cone boundaries. These topics deserve further numerical and experimental studies.

\section{Implications of fast ignition (FI) for inertial fusion energy power plants [71]}

Fast ignition offers a step-change in the pursuit of inertial fusion energy (IFE).

- Reduction in total driver energies, driver cost, and cost of electricity (COE) as well as reduction in radiation damage rates appear feasible.

- Possibility to use advanced target also documents as follows:

- reduce or eliminate need for breeding blankets

- exceptional safety and environmental characteristics.

- Relaxation of target fabrication requirements may also be envisioned. Fast ignition is expected to open the parameter space for innovation in chamber design (materials and configuration) and power conversion system design.

Fast ignition could be used with a variety of target designs.

- By moving from central, hot-spot ignition to fast ignition, the total driver energy falls from 3-5 MJ to less than 1 MJ.

- Even with added cost of ignition beam(s), FI might provide $30-40 \%$ reduction in capital cost.

- Expect similar reduction in COE.

- An attractive indirect driving scheme is thus featured in Figure 22. It highlights a two-sided geometry with the pellet compression driven from the right (here with a laser) and fast ignition coming from the left.

More specifically, Key et al. [72] have implemented above constraints (see Sect. 6) in Figure 23, at the scale of the two planned giant lasers LMJ (Bordeaux) and NIF (Livermore).

These authors consider a fuel density of $200 \mathrm{~g} \mathrm{~cm}^{-3}$ with $10 \%$ of the NIF beams adapted for chirped pulse amplification (CPA) [73]. Conversion at $60 \%$ efficiency to $0.53 \mu \mathrm{m}$ wavelength is assumed in order to help meet the $I \lambda^{2}$ constraint, giving a total short pulse energy of $200 \mathrm{~kJ}$. The beams would be focused to a clustered array of $25 \mu \mathrm{m}$ diameter spots at 20 ps pulse duration for $200 \mathrm{~g} \mathrm{~cm}^{-3}$ density; giving the $I \lambda^{2}$ value shown in Figure 1. It is further assumed that hole boring brings the ignitor beams to within about $100 \mu \mathrm{m}$ of the dense fuel where there is $30 \%$ conversion to electrons. Energy transfer by the electron beam to the ignition spark with self induced magnetic collimation [73] is assumed to be $66 \%$ efficient with a two fold increase in electron beam diameter between the optical focal spot and the ignition spark. Compression of the fuel is assumed to be with $8 \%$ hydrodynamic efficiency typical of direct drive, and with a ratio $\alpha=2$ of internal energy to the Fermi degenerate minimum. The gain is computed by specifying the fuel density and varying the fuel mass. Summing the required 
a)
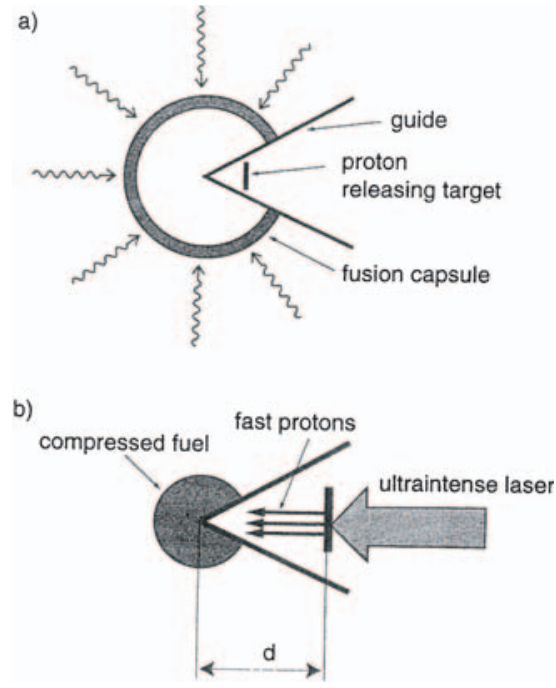

Fig. 21. Concept of conically guided target for fast ignition by laser accelerated protons: (a) target cross section; the fuel containing capsule is imploded by a suitable driver; (b) enlarged view of the central region around maximum fuel compression: at this time an ultraintense pulse is used to generate fast protons. The conical guide allows for placing the proton releasing target close to the compressed fuel (Atzeni et al. [70]).

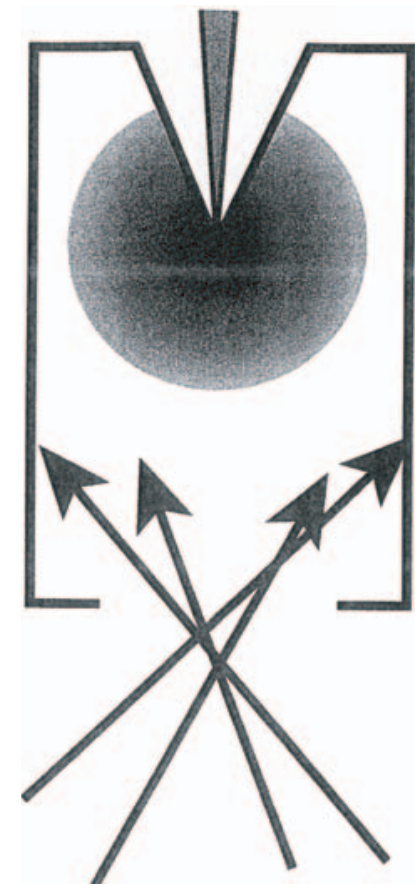

Fig. 22. Fast ignition target concept showing driver (right) and ignitor (left) beams. In this concept, plasma blowoff is excluded from the ignitor laser path and the driver lasers are concentrated at one end of the chamber. A similar scheme could be operated with intense and heavy ion beams in lieu of the driving laser. In this case, the laser entrance hole should be closed. primary laser energy delivered to ignitor beams and the laser energy needed for fuel compression gives the total laser energy input. The fusion burn output is calculated from the burn efficiency $\rho r /\left(\rho r+7 \mathrm{~g} \mathrm{~cm}^{-2}\right)$ and the fuel mass. For example, if $1 \mathrm{MJ}$ of laser energy is used to compress the fuel, the fuel mass is $4.1 \mathrm{mg}$ with a diameter of $340 \mu \mathrm{m}$, the $\rho r$ is $3.4 \mathrm{~g} \mathrm{~cm}^{-2}$ and the burn efficiency is 0.33 . The gain i.e. ratio of burn yield to laser energy is, therefore 330 .

It is necessary for obtaining high gain that the total laser driver energy be well above the ignition threshold and this sets a lower limit on the density as illustrated in Figure 23 because the threshold energy increases as $\rho^{-1.85}$. The upper density limit arises from $I \lambda^{2}$ in the focal spots, which increases approximately linearly with density as indicated in Figure 23. In this scenario there is an operating window centered at about $200 \mathrm{~g} \mathrm{~cm}^{-3}$.

The higher gain obtained in fast ignition arises because we assume a high compression efficiency $\eta=8 \%$, characteristic of direct drive, a low adiabat ratio $\alpha=2$ and a low density $\rho=200 \mathrm{~g} \mathrm{~cm}^{-3}$, where the energy used to compress the fuel dominates the total input energy and scales as $\eta \alpha \rho^{2 / 3}$. A low fuel adiabat is easier to achieve in fast ignition because the fuel density and pressure are reduced and there is no need for a central hot spot with its associated sensitivity to hydro-instabilities. Lower value of $\rho$ relative to direct drive is the primary source of the higher gain for FI shown in Figure 23.

This simple model has many limitations. The requirement in fast ignition research is to substantiate that the postulated behaviors can be obtained, in particular the efficient transfer of energy that if they can be obtained, then fast ignition has a unique capability for high gain of considerable potential importance for IFE.

High-yield D-T targets might then be affordable.

- In lieu of reducing the driver energy, one could choose to hold it constant and reach higher fusion yields.

- Repetition rates could be reduced from $\sim 5 \mathrm{~Hz}$ to only 1-2 Hz.

- Wider range of available target yields increases chamber design flexibility.

- For example, at low repetition rates, chamber clearing might be able to rely upon gravity clearing rather than forced flow schemes with a large pumping power.

- Tritium-lean targets would operate at high areal densities $\left(\rho r \sim 10-20 \mathrm{~g} / \mathrm{cm}^{2}\right)$ and have overall tritium percentages as low as $0.5 \%$ while managing a respectable gain and reducing significantly the burden on tritium inventory [74].

- Main fuel would be deuterium; sparkplug region, which the ignitor beam(s) would strike, would contain 20-50\% tritium.

- Due to high $\rho r$ and low tritium inventories, targets may be self-sufficient from tritium breeding perspective.

- Freed from need to keep blanket tritium breeding ratio above unity, one could select materials by other design criteria:

- Vapor pressure (affects beam propagation).

- Chemical compatibility. 


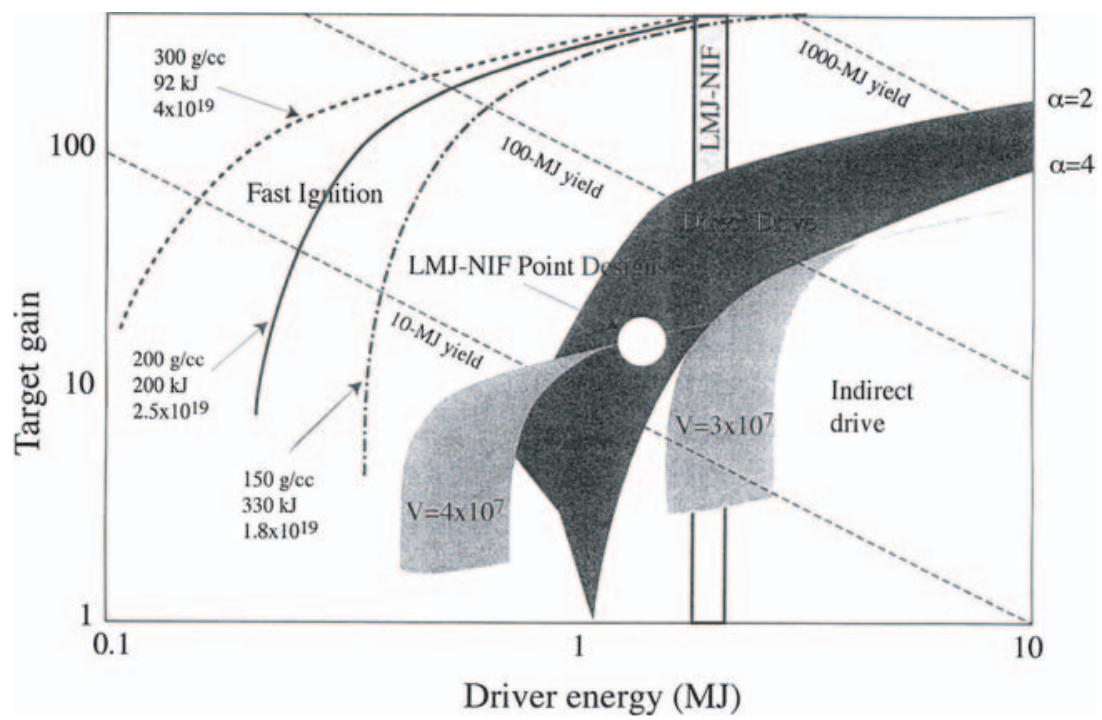

Fig. 23. Calculated gain curves for laser drivent ICF targets. Fast ignition plots are labeled with density, ignition spark driver energy and focal spot $l \lambda^{2}$. $V$ denotes pellet implosion velocity $(\mathrm{cm} / \mathrm{s})$ [72]

- Power conversion (higher temperature operation and/or direct conversion).

- Pumping power (important for liquid wall chambers).

- Coolant material abundance and cost.

- Safety and environment.

Fast ignition targets may be simpler and more robust.

- Since a central spark is not needed and shock waves need not propagate through the wall to the center, there is no problem with central mixing caused by a rough interior ice surface or with an asymmetric target.

- Might be able to 'hard-freeze' targets down to $4 \mathrm{~K}$, simplifying storage and injection.

- Alternate fuels with reduced cryogenic requirements might be possible; For example, $\mathrm{B}_{2} \mathrm{D}_{3} \mathrm{~T}_{3}$ melts above liquid nitrogen temperatures.

- Proposed areas of research are target design, target fabrication and injection, blanket engineering, and power plant design and economics.

\section{Frequently asked questions and conclusions}

In the present overview, we have essentially stressed the basic ideas and concepts of the novel compression possibilities afforded by the fast ignition scenario (FIS) [8]. The latter allows for a highly promising decoupling of the compression and ignition processes. As a result, the rather stringent requirements put on symmetry and ignition thresholds in the usual central hot spot ignition may be lavishly relaxed, as evidenced in Figure 23. We also emphasized the highly impressive experimental results achieved for the production of well collimated relativistic electron beams (REB) as well as the very interest- ing potential of the recently discovery of laser produced $\mathrm{MeV}$ protons.

It is nonetheless obvious that many hurdles still remain on the way to a successfull inertial pellet compression combined to an efficient fast ignition we highlight a few of them now along side with very recent encouraging technological perspectives.

How realistic is the REB penetration scenario, from the critical surface down to hot spot? (cf. Fig. 11).

The most critical issues involve understanding the physics of energy transport between the ignitor laser beam and the ignition spark. The photon pulse has to deliver up to hundreds kilojoules of energy in $\sim 10$ picoseconds in a spot a few $10 \mathrm{~s}$ of microns across located in the dense fuel. The intensities involved accelerate electrons to relativistic energies and lead to relativistic self-focusing of the laser beam, and the time involved is sufficient for the plasma structure and laser beam to co-evolve; there is much novel physics in this regime. The targeted ignition spot has a density much higher than the (relativistically corrected) critical density for transmission of light, and the energy of the laser is converted near that critical density surface to a directed jet of high energy electrons. The resulting electron currents greatly exceed the Alfven limit and require return current cancellation. The resulting EM fields modify the global electron flow, which is also subject to small scale Weibel instability. All of these phenomena have to be understood in some detail for success in fast ignition.

How intense proton beams in the $\mathrm{MeV}$ energy range react to electromagnetic instabilities? Preliminary investigations seem to indicate that those beams are immune to the Weibel instability, in the linear and quasi-linear (weakly turbulent) regimes, at least.

Is it possible to increase the laser production rate of $\mathrm{MeV}$ protons? Experimentalists feel reasonably optimistic about this issue and claim to be able to rise the given rate 
above 20 per cent, within a five years period, from now. Then, a proton induced FIS could be envisioned.

Does it make sense to expect much more energetic REB from PW lasers operating in the $10^{23}$ $10^{24} \mathrm{w} / \mathrm{cm}^{2}$ irradiance regime? Yes, it does, several groups are indeed presently active in this field. So intense REB with energies in the hundreds $\mathrm{MeV}$ might be considered. Then, substantial modifications to their transport scenarii, according to the suggestions of reference [47], could be of relevance. As far as the diversion of REB kinetic energy through plasma modes is concerned, the emphasis could then be switched from Weibel to Langmuir instabilities.

How deep is the present understanding of the stopping of intense charged particle beams through collective modes excited in an overdence target plasma with a strong density gradient?

A lot of theoretical as well as numerical insights have already been gathered in this area of endeavor. Numerical simulations of the nonlinear WEI have already been documented above. Here, we intend to stress that many Russian theory groups have produced analytic investigations of the highest quality (see for instance $[75,76]$ ) underlying some of the more illustrative PIC simulations, in particular those led by Pukhov [40,44] and Ruhl [34,45].

What is the present status of the hole boring process?

As far as present REB-cold target interaction experiments are concerned, it appears well documented. However, some PIC simulations of precompressed fuels have recently demonstrated that this compulsory predecessor of FIS does not persist for target layers with a density above $40 n_{c}$. These controversial results have thus led some authors [77] to question the whole fast ignition scenario and replace it with a volume ignition one which is intrinsically less efficient from the energy point of view.

This situation explains that many researchers in the field would select the wedged (cone angle) approach as the most appropriate for achieving a full (see Sect. 4) fledged FIS. The wedged procedure allows to shortcut most of the uncertainties attached to the REB propagation in the outer layers of the compressed DT core.

How reliable is the wedged approach?

Up to now, experiments performed by the BritischJapanese team (Kodama et al. [48]) have confirmed every positive expectation of that scheme, albeit at a rather modest ignition energy level $(\sim 60 \mathrm{~J})$.

Moreover, the latter appears less demanding in symmetry request during the compression phase. Nonetheless, the spurious outputs due to a mixing zone between the cone heavy material with the fuel should be carefully investigated. Ongoing spectroscopic investigations of this issue should allow to settle positively the matter.

How to get efficiently and rapidly introduced to the proliferating literature on fast ignition? I hope that the present overview will efficiently contribute to that goal, the list of published proceedings of international workshops dedicated to this presently very hot topic is given in the reference [78-80].
In the near future, increased PW-laser facilities as well as still increasing CPU computer resources are expected to clear up many of the afore mentioned issues.

Up to now, FIS features the best promises of success for the whole inertial fusion energy program. It can be easily adapted to any possible compression driver: laser, heavy ion beams or Z-pinch and removes most of the extreme requirements demanded by the standard scenario based on central spot ignition.

\section{References}

1. J.D. Lindl, Phys. Plasmas 2, 3933 (1995) and also M. André, C. Cavailler, F. Jequier, Le Vide 307, 13 (2003)

2. S.E. Bodner, Phys. Rev. Lett. 33, 761 (1974)

3. R.D. Richtmyer, Commun. Pure Appl. Math. 13, 297 (1960) and E.E. Meshkov, Fluid Dynam. 4, 101 (1969)

4. H. Takabe, et al., Phys. Fluids 26, 2299 (1983)

5. E. Storm, et al., Progress in laboratory high gain ICF: prospects for the future, UCRL-99427 (1988)

6. H. Azechi, et al., Laser Part. Beams 9, 193 (1991)

7. J.M. Soures, et al., Phys. Plasmas 3, 2108 (1996)

8. M. Tabak, et al., Phys. Plasmas 1, 1626 (1994)

9. J. Meyer-ter-Vehn, Plasma Phys. Control. Fusion 43, A113 (2001)

10. B. Luther-Davies, et al., Laser Phys. 1, 325 (1991)

11. J. Meyer-ter-Vehn, Nucl. Fusion 22, 531 (1982)

12. R. Kidder, Nucl. Fusion 16, 405 (1976)

13. S. Atzeni, Jpn J. Appl. Phys. 34, 1980 (1995)

14. C. Deutsch, et al., Phys. Rev. Lett. 77, 2483 (1996); 85, 1140 (E) (2000)

15. For instance see the review D. Umstadter, Phys. Plasmas 8, 1774 (2001); ibid, J. Phys. D: Appl. Phys. 36, R151 (2003)

16. E. Lefebvre, G. Bonnaud, Phys. Rev. Lett. 74, 2002 (1995)

17. J.C. Adam, et al., Phys. Rev. Lett. 84, 598 (2000); J.C. Adam, et al., Phys. Rev. Lett. 78, 4765 (1997)

18. R.B. Palmer, Part. Accel. 11, 81 (1980)

19. K.B. Wharton, et al., Phys. Rev. Lett. 81, 822 (1998)

20. M.H. Key, et al., Phys. Plasmas 5, 1966 (1998)

21. S. Bastiani, et al., Phys. Rev. E 56, 7179 (1997)

22. J. Fuchs, et al., Phys. Plasmas 6, 2569 (1999)

23. Th. Schlegel, et al., Phys. Rev. E 60, 2209 (1999)

24. U. Teubner, et al., Phys. Rev. E 54, 4167 (1996)

25. M. Roth, et al., in Inertial Fusion Sciences and Application 99, edited by Ch. Labaune, W.J. Hogan, K.A. Tanaka (Elsevier, Paris, 2000)

26. G. Malka, J.L. Miquel, Phys. Rev. Lett. 77, 75 (1996)

27. S.C. Wilks, et al., Phys. Rev. Lett. 69, 1383 (1992)

28. A.J. Mackinnon, et al., Phys. Plasmas 6, 2185 (1999)

29. M. Borghesi, et al., Phys. Rev. Lett. 80, 5137 (1998)

30. M. Tatarakis, et al., Phys. Rev. Lett. 81, 999 (1998); K. Krushelnik, et al., Phys. Rev. Lett. 78, 4047 (1997)

31. M. Borghesi, et al., Phys. Rev. Lett. 83, 4309 (1999)

32. L. Gremillet, et al., Phys. Rev. Lett. 83, 5015 (1999)

33. P.A. Norreys, et al., Phys. Plasmas 6, 2150 (1999) and also PIC simulations in G.A. Askar'yan, S.V. Bulanov, et al., Plasma Phys. Controlled Fusion 39, 137 (1997)

34. H. Ruhl, et al., Phys. Rev. Lett. 82, 743746 (1998)

35. Y. Sentoku, et al., Phys. Plasmas 6, 2855 (1999)

36. F. Brunel, Phys. Rev. Lett. 59, 52 (1987) 
37. D. Gordon, et al., Phys. Rev. Lett. 80, 2133 (1998)

38. W.L. Kruer, The physics of Laser Plasmas Interactions (Addison-Wesley, Redwood City, CA, 1988)

39. E. Lefebvre, et al., Phys. Rev. E 55, 1011 (1997)

40. A. Pukhov, J. Meyer-ter-Vehn, Phys. Rev. Lett. 79, 2686 (1997) and also A. Pukhov, et al., Phys. Plasmas 5, 1880 (1998)

41. W. Yu, et al., Phys. Rev. Lett. 85, 570 (2000)

42. M. Reiser, Theory and design of charged particle beams (John Wiley and Sons, Inc., New York, 1994), p. 6

43. E.S. Weibel, Phys. Rev. Lett. 2, 83 (1959)

44. M. Honda, A. Pukhov, J. Meyer-ter-Vehn, Phys. Rev. Lett. 85, 2118 (2000); M. Honda, Phys. Plasmas 7, 1606 (2000)

45. H. Ruhl, et al., Plasma Phys. Rep. 27, 387 (2001) and also H. Ruhl, Plasma Sources Sci. Technol. 11, A-154 (2002) and also M.N. Naumova, S. Bulanov, T.Z. Esirkepov, et al., Phys. Rev. Lett. 87, 185004 (2001)

46. G. Logan, Garching Fast Ignitor Workshop, Sept. 17-19 (1997)

47. V.M. Malkin, N.J. Fish, Phys. Rev. Lett. 89, 125004 (2002)

48. R. Kodama, et al., Nature 412, 799 (2001); 418, 933 (2002)

49. M.H. Key, Nature 412, 775 (2001)

50. G. Yonas, IEEE Trans. Nucl. Sci. NS-26, 610 (1979)

51. S.C. Wilks, Phys. Fluids B5, 2603 (1993)

52. V.V. Val'chuck, et al., Plasma Phys. Rep. 21, 159 (1995)

53. D. Bohm, D. Pines, Phys. Rev. 85, 338 (1952)

54. C. Deutsch, et al., Nucl. Instrum. Methods Phys. Res., Sect. A 278, 38 (1989)

55. H.H. Hubbel, R.D. Birkoff, Phys. Rev. A 26, 2460 (1982)

56. P.C. Hemmer, I.E. Farquahr, Phys. Rev. 168, 294 (1968)

57. M. Busquet, Chocs 13, 57 (1995)

58. C. Deutsch, P. Fromy, Phys. Plasmas 6, 3587 (1999) and Phys. Rev. E 61, 4322 (2000)

59. S. Atzeni, Phys. Plasmas 6, 3316 (1999); A.R. Piriz, M.M. Sanchez, Phys. Plasmas 5, 4373 (1998) which predict ignition with 10 times less energy than S. Atzeni

60. E.L. Clark, et al., Phys. Rev. Lett. 84, 670 (2000)

61. A. Maksimchuk, et al., Phys. Rev. Lett. 84, 4108 (2000); K. Nomoto, et al., Appl. Phys. Lett. 78, 595 (2001)

62. R.A. Snavely, et al., Phys. Rev. Lett. 85, 2945 (2001)
63. S.P. Hatchett, et al., Phys. Plasmas 7, 2076 (2000)

64. S.C. Wilks, A.B. Langdon, et al., Phys. Plasmas 8, 542 (2001)

65. M. Roth, et al., Phys. Rev. Lett. 86, 436 (2001).

66. M. Borghesi, et al., Laser Part. Beams 20, 31 (2002)

67. W.L. McLaughlin, et al., Nucl. Instrum. Methods Phys. Res. A 302, 165 (1991)

68. W. Enge, Rad. Meas. 25, 11 (1995)

69. A.J. McKinnon, et al., Phys. Rev. Lett. 86, 1769 (2001)

70. S. Atzeni, M. Temporal, J.J. Honrubbia, Nucl. Fusion 42, L1 (2002) and also M. Temporal, J.J. Honrubbia, S. Atzeni, Phys. Plasmas 9, 3098 (2002)

71. S.P. Hatchett, M. Tabak, R. Turner, R. Stephens, Proceedings 28th European Physical Society Conference on Controlled Fusion and Plasma Physics and 5th Workshop on Fast Ignition of Fusion Targets, Funchal 2001, edited by C. Silva, C. Varandas, D. Campbell (European Physical Society, Mulhouse, 2001) Europhys. Conf. Abstr., Vol. 25A, paper W. 18, p. 33 (workshop section); J.F. Latkowski, et al., Lawrence Livermore National Laboratory report (2000)

72. M.H. Key, et al., J. Fusion Energy 17, 231 (1998)

73. M.D. Perry, G. Mourou, Science 264, 017 (1994)

74. G.H. Miley, Laser Int. Rel. Phen. 5, 313 (1981); M. Tabak, Nucl. Fusion 36, 147 (1996); S. Atzeni, M.L. Ciampi, Nucl. Fusion 37, 1665 (1997); N.A. Tahir, D.H.H. Hoffmann, Nucl. Instrum. Methods A 415, 151 (1998)

75. S.V. Bulanov, et al., Phys. Lett. A 195, 84 (1994); Phys. Rev. Lett. 74, 710 (1995)

76. G.A. Askar'yan, et al., JETP Lett. 60, 241 (1994)

77. 2nd Topical Workshop on fast ignition, edited by J. Meyer-ter-Vehn, MPQ report 226, Garching, Sept. 1997 and also S. Hain, P. Muelser, Phys. Rev. Lett. 86, 1015 (2001)

78. 3rd International Workshop on the Fast Ignition of Fusion Targets, edited by P.A. Norreys, RAL-TR-1998085, Rutherford-Appleton Lab. 8/11/1999

79. 4th International Workshop on Fast Ignition, edited by P. Mora, J.C. Gauthier, École Polytechnique, 7/4/2000

80. Proceed. 2nd International Conference on inertial fusion sciences and applications (IFSA2001), Kyoto (Elsevier, Paris, 2002) 